This is a pre-copyedited, author-produced PDF of an article accepted for publication in Geophysical Journal International following peer review. The version of record 'Santiago G. Solazzi, Simón Lissa, J. Germán Rubino, Klaus Holliger, Squirt flow in partially saturated cracks: A simple analytical model, Geophysical Journal International, 2021;, ggab249', is available online at: https://doi.org/10.1093/gji/ggab249.

submitted to Geophys. J. Int.

\title{
Squirt flow in partially saturated cracks: A simple
}

\section{analytical model}

\author{
Santiago G. Solazzi ${ }^{1}$, Simón Lissa ${ }^{1}$, J. Germán Rubino ${ }^{2}$, and Klaus Holliger ${ }^{1}$ \\ ${ }^{1}$ Institute of Earth Sciences, University of Lausanne, Lausanne, Switzerland. \\ ${ }^{2}$ CONICET, Centro Atómico Bariloche - CNEA, San Carlos de Bariloche, Argentina.
}

\section{SUMMARY}

Obtaining the seismic response of rocks containing cracks whose scales are much smaller than the prevailing wavelengths is a classic and important problem in rock physics. Seminal analytical models yield the seismic signatures of cracked rocks saturated with a single fluid phase. However, in a wide variety of practically relevant scenarios, cracks may be partially saturated with multiple immiscible fluids of contrasting compressibilities, such as gas and water. When a passing seismic wave deforms the medium, fluid pressure gradients arise within such partially saturated cracks, which, in turn, tend to relax through a process commonly known as squirt flow. The corresponding viscous dissipation may greatly affect the seismic amplitudes and velocities, as well as the anisotropic behaviour of the medium. To date, extensions of classical analytical models to include squirt flow occurring within isolated partially saturated cracks remain limited either in the saturation or in the frequency range. In this work, we present a simple analytical model to compute the seismic response of rocks containing partially saturated aligned cracks accounting for squirt flow effects. First, we solve the linearised Navier-Stokes equations within a partially saturated penny-shaped crack subjected to an oscillatory strain. Then, we obtain a closed analytical expression for a complex-valued frequency-dependent effective fluid bulk modulus which accounts for the stiffness variations of each crack due to squirt flow. 
Using classic effective medium models, together with such an effective saturating fluid, we retrieve the effective compliance matrix of the probed partially saturated cracked rock. The proposed analytical solution is validated by comparison with corresponding 3D numerical simulations and existing analytical models.

Key words: Seismic attenuation; Seismic anisotropy; Acoustical properties; Wave propagation

\section{INTRODUCTION}

Determining the fluid content of geological formations from seismic data is a complex task of great importance in a number of pertinent scenarios, such as, oil and gas exploration and production, geothermal energy exploitation, $\mathrm{CO}_{2}$ geosequestration, and enhanced oil recovery operations. Evidence shows that, seismic waves travelling in partially saturated formations can suffer from large attenuation and phase velocity dispersion due to fluid pressure diffusion (FPD) processes (e.g., Cadoret et al. 1998; Yin et al. 1992; Tisato \& Quintal 2013; Chapman et al. 2016). In addition, the mere presence of cracks, which are largely recognised as omnipresent in Earth's crust materials, can also affect the seismic amplitudes and velocities, even if their characteristic scales are much smaller than the prevailing seismic wavelengths (e.g., Hudson 1980; Cheng 1993). In this context, models addressing the interrelationships existing between crack presence, pore fluid content, and corresponding seismic signatures, are of particular value for exploring partially saturated geological formations.

Obtaining the seismic response of a rock containing cracks that are much smaller than the seismic wavelengths is a classic and practically important problem in rock physics (e.g., Garbin \& Knopoff 1973; Budiansky \& O'Connell 1976). This can be achieved by deriving the effective elastic constants of a homogeneous solid which exhibits the same velocity variations with direction as the cracked solid. Most of the existing models are based on the classic work of Eshelby (1957), who derived the elastic response of a solid comprising an elliptical inclusion under a load applied at a large distance. Crampin (1978) obtained effective elastic constants by modelling the variation of seismic velocity through an isotropic solid comprising a low concentration of parallel cracks using the model of Garbin \& Knopoff $(1973,1975 b, a)$. Later on, Hudson $(1980,1981)$ developed a more general theoretical approach for evaluating the effective elastic constants of cracked solids. Subsequently, other authors have made modifications to Hudson's solution. For example, Cheng (1993) extended the restrictions of Hudson's model with regard to the aspect ratio of the cracks. However, the model developed by Hudson (1981) constitutes arguably one of the most employed frameworks for elastic constant determination of elastic 
isotropic media containing a dilute concentration of penny-shaped cracks. This model considers that cracks are in the so-called unrelaxed state and, thus, FPD processes triggered by seismic waves are not accounted for (e.g., Mavko et al. 2009). The corresponding effective elastic constant in the relaxed state can be obtained by considering dry cracks and, then, using the anisotropic Gassmann (1951) equations to saturate them. In the case of partial saturation, the latter is done employing an effective pore fluid (e.g., Mavko et al. 2009). However, extending this theory to the partially relaxed state and, thus, to the entire frequency band, requires a more comprehensive approach.

There is increasing experimental evidence indicating that squirt flow at the microscopic/mesoscopic scale may be largely responsible for the observed attenuation and phase velocity dispersion of seismic waves in the exploration seismic frequency range (e.g., Subramaniyan et al. 2015; Chapman et al. 2019). Squirt flow models assume that the passage of a seismic wave can induce pressure gradients between hydraulically connected regions of the medium presenting contrasting compressibilities, which, in turn, relax trough FPD (see Müller et al. 2010, for a comprehensive review). Several classic works in the literature addressed the problem of seismic attenuation and dispersion due to squirt flow occurring within cracks saturated with two immiscible fluid phases of contrasting compressibilities, which are classically classified as wetting (i.e., water) and non-wetting (i.e., gas, oil) in relation to their capacity to wet the cracks' walls. Mavko \& Nur (1979) developed expressions for the attenuation of Pand S-waves in a material with partially saturated cracks with random orientations based on a 2D parallel walled pore geometry. Palmer \& Traviolia (1980) extended the model of Mavko \& Nur (1979) to 3D contacts between individual grains. However, the models of Palmer \& Traviolia (1980) and Mavko \& Nur (1979) are both restricted to low wetting-fluid phase saturations and disregard the compressibility of the non-wetting fluid as well as the dissipative effects associated with the flow of this fluid phase. Hudson (1988) developed an extension of his classic penny-shaped model to approximate the effects of FPD within partially saturated cracks embedded in an impermeable background. This model was later on improved by Pointer et al. (2000) to account for the effects of the non-wetting phase compressibility. However, the resulting P- and S-wave attenuation estimates are valid only at low frequencies, in particular, they present a linear behaviour with regard to frequency and wave speeds are non-dispersive. Murphy et al. (1986) presented a solution for the squirt flow phenomenon, considering a compliant flat crack connected to a stiff toroidal pore, which included a saturation term, thus, permitting the length of the wetting fluid droplet to vary within the crack. However, the toroidal pore is not permitted to deform under external or internal loading and the compressibility of the non-wetting fluid in the crack is, once again, disregarded. Later, Walsh (1995) addressed the effects of a fluid lens within a crack on seismic attenuation. For this, the oscillations in the crack aperture resulting from the passage of a seismic wave where evaluated by considering a zero pressure condition at the boundaries 
of the wetting fluid lens, thus assuming a zero bulk modulus for the non-wetting phase. To sum up, as far as the authors know, the available models addressing squirt flow effects in partially saturated cracks exhibit one or more of the following limitations: (i) The model cannot represent the corresponding mechanism across the entire frequency band (e.g., Hudson 1988; Pointer et al. 2000); (ii) the model is restricted with regard to the saturation range (e.g., Mavko \& Nur 1979; Hudson 1988); (iii) the formulation assumes that the non-wetting fluid phase does not play a significant role in the squirt flow process (Murphy et al. 1986; Walsh 1995). Furthermore, all previous studies disregard the effects of capillary forces in the squirt flow mechanism.

In this work, we present a new analytical model to account for squirt flow effects in rocks containing aligned partially saturated cracks embedded in an elastic isotropic background for the full range of saturations and frequencies. For this, we consider that the aligned penny-shaped cracks undergo an oscillatory compression triggered by a passing wave. Each crack is saturated with a non-wetting phase bubble, located at the cracks' centre, which is surrounded by wetting phase fluid. The analysis accounts for the compressibilities of both saturating fluid phases and, also, for capillary effects. We derive an expression for the complex-valued and frequency-dependent bulk modulus of an effective fluid, which yields the stiffness variations of the partially saturated cracks with frequency due to internal FPD. Such an effective fluid is then employed as saturating fluid within Hudson's (1981) model to obtain the complex-valued and frequency-dependent components of the effective compliance matrix of the vertical transversely isotropic (VTI) medium. The latter allows to compute the angle-, saturation, and frequency-dependent seismic velocities and quality factors of the corresponding medium. The proposed analytical solution is validated by comparing the resulting velocity dispersion and attenuation estimates with those provided by Pointer's (2000) low-frequency solution and by finite element numerical simulations (Quintal et al. 2019), which solve the FPD problem using the quasi-static and linearised coupled version of the Lamé-Navier and Navier-Stokes equations in a 3D cracked medium.

\section{THEORETICAL DESCRIPTION OF THE MODEL}

\subsection{Effective stiffness of a partially saturated penny-shaped crack}

Let us consider a rock composed of an isotropic and homogeneous elastic background with bulk modulus $K_{s}$ and shear modulus $\mu_{s}$, permeated by a set of aligned penny-shaped cracks with radius $r_{c}$ and a thickness $h_{0}$ (Fig. 1a). As previously mentioned, cracks are saturated with two immiscible fluid phases of contrasting compressibilities. Following Mavko \& Nur (1979), we assume that the non-wetting phase is located at the cracks' center, forming a bubble of radius $r_{b}$, and the wetting fluid phase saturates the remaining ring-shaped region (Figs $1 \mathrm{~b}$ and 1c). The effective stiffness of a single 


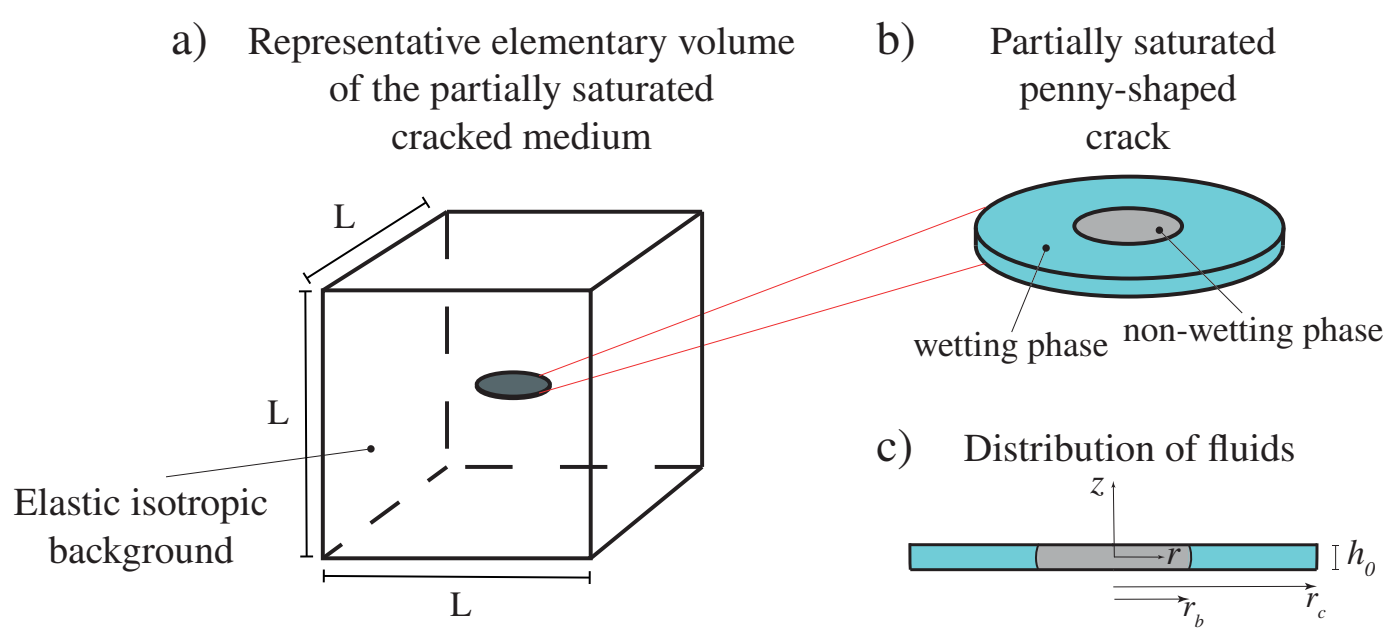

Figure 1. (a) Sketch of a representative elementary volume (REV) of the explored cracked medium and (b) blowup of a corresponding partially saturated crack. The central volume (grey) denotes the location of the non-wetting fluid phase and the remaining region (blue) denotes that of the wetting phase. (c) Vertical profile, illustrating the distribution of the fluid phases within the crack.

crack $K^{*}$ is given by the ratio of the force $\Delta F$ exerted by the fluid onto the crack walls in response to a uniaxial dynamic loading displacement $-\Delta h$ (e.g., Murphy et al. 1986; Gurevich et al. 2010)

$$
K^{*}=-\frac{\Delta F}{\Delta h} \text {. }
$$

The force $\Delta F$, referred to as acoustic load in the work of Murphy et al. (1986), is given by the integral of (i) the non-wetting fluid pressure $p_{\mathrm{n}}$ over the area occupied by this phase $A_{\mathrm{n}}$ and (ii) the wetting fluid pressure $p_{\mathrm{w}}$ over the remaining area $A_{\mathrm{w}}$,

$$
\Delta F=\int_{A_{\mathrm{n}}} p_{\mathrm{n}}(r) \mathrm{d} A_{\mathrm{n}}+\int_{A_{\mathrm{w}}} p_{\mathrm{w}}(r) \mathrm{d} A_{\mathrm{w}}
$$

where the subscripts $\mathrm{n}$ and $\mathrm{w}$ refer to the non-wetting and wetting fluid phases, respectively.

The wetting phase saturation $S_{\mathrm{w}}$ is given by

$$
S_{\mathrm{w}}=\frac{V_{\mathrm{w}}}{V_{\text {crack }}}=\frac{\left(r_{c}^{2}-r_{b}^{2}\right)}{r_{c}^{2}}
$$

with $V_{\mathrm{w}}$ the volume occupied by wetting phase and $V_{\text {crack }}$ the crack's volume. The non-wetting fluid phase saturation responds to $S_{\mathrm{n}}=1-S_{\mathrm{w}}=r_{b}^{2} / r_{c}^{2}$.

\subsection{Equations of motion and continuity}

Following Gurevich et al. (2010), we model the squirt flow process by solving the Navier-Stokes equations in the crack gap under the following assumptions: (i) no-slip conditions between the fluid and the crack walls; (ii) radial flow (uni-dimensional in cylindrical coordinates); (iii) small deformations 
(i.e., we drop convective terms); and (iv) quasi-static conditions (i.e, we drop acceleration terms). The corresponding equation of motion of a fluid within the crack reduces to (e.g., Murphy et al. 1986)

$$
W=-D_{0}^{-1} \frac{\partial p_{f}}{\partial r}
$$

where $p_{f}$ denotes the pore fluid pressure perturbation and $W=v_{r} h_{0}$ is the linearised sectional volume flow rate, with $v_{r}$ being the radial velocity averaged over the crack section and $h_{0}$ the unperturbed aperture of the crack. Also, $D_{0}=12 \eta_{f} / h_{0}^{3}$, where $\eta_{f}$ is the fluid shear viscosity.

The continuity equation for the fluid is classically given by (e.g., Murphy et al. 1986)

$$
\frac{\partial\left(\rho_{f} h\right)}{\partial t}+\nabla \cdot\left(\rho_{f} h \mathbf{v}\right)=0
$$

where $\mathbf{v}$ is the fluid velocity vector, $h=h_{0}-\Delta h$, and $\rho_{f}$ is the fluid density. Eq. (5) can be further simplified by considering the above mentioned assumptions together with: (i) small density variations along the radial axis and (ii) adiabatic conditions, which are common assumptions in the context of seismic wave propagation (e.g., Bourbié et al. 1987). In this context, Eq (5) in cylindrical coordinates reduces to (e.g., Murphy et al. 1986)

$$
\frac{\partial h}{\partial t}+\frac{h_{0}}{K_{f}} \frac{\partial p_{f}}{\partial t}+\frac{\partial W}{\partial r}=-\frac{W}{r}
$$

where $K_{f}=\rho_{f}\left(\partial p_{f} / \partial \rho_{f}\right)$ is the bulk modulus of the saturating fluid.

Combining Eqs (4) and (6), and considering time-harmonic $e^{i \omega t}$ behaviour, we obtain the following expression in the space-frequency domain (e.g., Gurevich et al. 2010)

$$
\frac{\partial p_{f}}{\partial r}+\frac{1}{r} \frac{\partial p_{f}}{\partial r}+k_{f}^{2} p_{f}=C
$$

where $k_{f}^{2}=-i \omega D_{0} h_{0} / K_{f}$ and $C=i \omega D_{0} \Delta h$. This equation, which is assumed to hold for both the wetting and the non-wetting phases within a partially saturated crack, is the main expression to be solved in this work. The fluid pressures for the saturating fluids, for a given angular frequency $\omega$, respond to

$$
p_{f}(r)= \begin{cases}p_{\mathrm{n}}(r), & \text { for } r=\left[0, r_{b}\right], \\ p_{\mathrm{w}}(r), & \text { for } r=\left[r_{b}, r_{c}\right] .\end{cases}
$$

The general solution of Eq. (7) is a combination of zero-order Bessel functions $J_{0}$ and $Y_{0}$ of the first and second kind, respectively (e.g., Abramowitz \& Stegun 1965; Murphy et al. 1986):

$$
\begin{array}{lll}
p_{\mathrm{n}}(r)= & \mathcal{A}_{\mathrm{n}} J_{0}\left(k_{\mathrm{n}} r\right)+\mathcal{B}_{\mathrm{n}} Y_{0}\left(k_{\mathrm{n}} r\right)+\frac{C_{\mathrm{n}}}{k_{\mathrm{n}}^{2}}, & \text { for } r=\left[0, r_{b}\right], \\
p_{\mathrm{w}}(r)=\mathcal{A}_{\mathrm{w}} J_{0}\left(k_{\mathrm{w}} r\right)+\mathcal{B}_{\mathrm{w}} Y_{0}\left(k_{\mathrm{w}} r\right)+\frac{C_{\mathrm{w}}}{k_{\mathrm{w}}^{2}}, & \text { for } r=\left[r_{b}, r_{c}\right],
\end{array}
$$

where $\mathcal{A}_{f}$ and $\mathcal{B}_{f}$, with $f=\mathrm{w}$, n, are four integration constants whose expressions are obtained from the boundary conditions of the problem, which are detailed in the next section. 


\subsection{Boundary conditions}

In order to obtain the pressures of both fluid phases (Eqs 9 and 10) and, through them, derive an expression for the effective crack stiffness (Eq. 1), we need a set of suitable boundary conditions. We require: (i) a non-diverging (finite-value) pressure at the center of the crack; (ii) a no-flow condition at the edges of the crack; as well as (iii) mass conservation and (iv) continuity of pressures at the interface between fluids.

The first condition, states that the pressure value at $r=0$ must be finite. Given that second-kind Bessel functions present a singularity at the origin, that is, $\lim _{r \rightarrow 0} Y_{0}\left(k_{\mathrm{n}} r\right)=\infty$, the $B_{\mathrm{n}}$ term has to be null for the pressure to be non-diverging in Eq. (9). Conversely, the no-flow condition in the crack edges implies that $\left.W\right|_{r_{c}}=0$, which, considering Eq. (4), reduces to

$$
\left.\frac{\partial p_{\mathrm{w}}(r)}{\partial r}\right|_{r_{c}}=0
$$

Mass conservation and pressure equilibrium conditions must hold at the interface between fluids. On the one hand, mass conservation is preserved by imposing continuity of the sectional volume-flow rates at the interface, located at $r_{b}$,

$$
\left.W_{\mathrm{n}}\right|_{r_{b}}=\left.W_{\mathrm{w}}\right|_{r_{b}}
$$

Considering Eq. (4), this boundary condition reduces to

$$
\left.\frac{1}{D_{0, \mathrm{n}}} \frac{\partial p_{\mathrm{n}}(r)}{\partial r}\right|_{r_{b}}=\left.\frac{1}{D_{0, \mathrm{w}}} \frac{\partial p_{\mathrm{w}}(r)}{\partial r}\right|_{r_{b}}
$$

On the other hand, pressure equilibrium must hold at the interface between wetting and non-wetting fluid phases. For this, it is important to recall that a pressure jump exists between both saturating phases, which is equilibrated by the action of the capillary pressure. In a state of equilibrium, the capillary pressure responds to the Young-Laplace equation (e.g., Blunt 2017)

$$
\left.\bar{p}_{\mathrm{n}}(r)\right|_{r_{b}}-\left.\bar{p}_{\mathrm{w}}(r)\right|_{r_{b}} \simeq \frac{2 \sigma \cos \gamma}{h_{0}}
$$

where $\sigma$ denotes the interfacial tension, $\gamma$ the contact angle, and $\bar{p}_{i}$ with $i=\mathrm{w}, \mathrm{n}$ being the unperturbed state of the corresponding fluid pressures, that is, prior to the seismic perturbation. Eq. (14) implies that $\bar{p}_{\mathrm{n}}>\bar{p}_{\mathrm{w}}$. This pressure build-up balances the action of capillary tension, which drives water to further wet the crack. Note that the pore fluid pressures $p_{\mathrm{n}}(r, \omega)$ and $p_{\mathrm{w}}(r, \omega)$ considered in our model are perturbations with respect to their steady-state counterparts $\bar{p}_{\mathrm{n}}$ and $\bar{p}_{\mathrm{w}}$. Then, we can 
rewrite Eq. (14) in the perturbed state as

$$
\begin{aligned}
\left.\left(\bar{p}_{\mathrm{n}}+p_{\mathrm{n}}\right)\right|_{r_{b}}-\left.\left(\bar{p}_{\mathrm{w}}+p_{\mathrm{w}}\right)\right|_{r_{b}} & =\frac{2 \sigma \cos \gamma}{h}, \\
\left.p_{\mathrm{n}}\right|_{r_{b}}-\left.p_{\mathrm{w}}\right|_{r_{b}} & =\frac{2 \sigma \cos \gamma}{h}-\left.\left(\bar{p}_{\mathrm{n}}-\bar{p}_{\mathrm{w}}\right)\right|_{r_{b}}, \\
\left.p_{\mathrm{n}}\right|_{r_{b}}-\left.p_{\mathrm{w}}\right|_{r_{b}} & =\frac{2 \sigma \cos \gamma}{\left(h_{0}-\Delta h\right)}-\frac{2 \sigma \cos \gamma}{h_{0}}, \\
\left.p_{\mathrm{n}}\right|_{r_{b}}-\left.p_{\mathrm{w}}\right|_{r_{b}} & =2 \sigma \cos \gamma \frac{\Delta h}{h_{0}^{2}\left(1-\Delta h / h_{0}\right)} .
\end{aligned}
$$

Considering that $\Delta h / h_{0}<<1$, we approximate the boundary condition for the pressures at the fluid interface as

$$
\left.p_{\mathrm{n}}\right|_{r_{b}}-\left.p_{\mathrm{w}}\right|_{r_{b}}=\frac{2 \sigma \cos \gamma}{h_{0}^{2}} \Delta h
$$

For simplicity, $\gamma$ is taken as a constant parameter and, thus, processes such as contact angle hysteresis and contact line movements are not accounted for in our model (e.g., Rozhko \& Bauer 2019).

\subsection{Solution to the problem}

The pore fluid pressures of the wetting and non-wetting phases are obtained from Eqs (9) and (10) and the above described boundary conditions. The given formulation yields closed analytical expressions for the wetting and non-wetting fluid pressures as functions of the radius

$$
\begin{aligned}
& p_{\mathrm{w}}(r)=\mathcal{B}_{\mathrm{w}}^{*} \frac{\Delta h}{h_{0}} T_{0}(r)+\frac{C_{\mathrm{w}}}{k_{\mathrm{w}}^{2}}, \\
& p_{\mathrm{n}}(r)=\mathcal{A}_{\mathrm{n}}^{*} \frac{\Delta h}{h_{0}} J_{0}\left(k_{\mathrm{n}} r\right)+\frac{C_{\mathrm{n}}}{k_{\mathrm{n}}^{2}},
\end{aligned}
$$

with

$$
\begin{aligned}
T_{0}(r) & =\left(Y_{0}\left(k_{\mathrm{w}} r\right)-\frac{Y_{1}\left(k_{\mathrm{w}} r_{c}\right)}{J_{1}\left(k_{\mathrm{w}} r_{c}\right)} J_{0}\left(k_{\mathrm{w}} r\right)\right) \\
\mathcal{B}_{\mathrm{w}}^{*} & =\left(T_{0}\left(r_{b}\right)-\sqrt{\frac{\eta_{\mathrm{n}} K_{\mathrm{n}}}{\eta_{\mathrm{w}} K_{\mathrm{w}}}} \frac{J_{0}\left(k_{\mathrm{n}} r_{b}\right)}{J_{1}\left(k_{\mathrm{n}} r_{b}\right)} T_{1}\left(r_{b}\right)\right)^{-1}\left(K_{\mathrm{w}}-K_{\mathrm{n}}-\frac{2 \sigma \cos \gamma}{h_{0}}\right) \\
T_{1}(r) & =\left(Y_{1}\left(k_{\mathrm{w}} r\right)-\frac{Y_{1}\left(k_{\mathrm{w}} r_{c}\right)}{J_{1}\left(k_{\mathrm{w}} r_{c}\right)} J_{1}\left(k_{\mathrm{w}} r\right)\right), \\
\mathcal{A}_{\mathrm{n}}^{*} & =\mathcal{B}_{\mathrm{w}}^{*} \sqrt{\frac{\eta_{\mathrm{n}} K_{\mathrm{n}}}{\eta_{\mathrm{w}} K_{\mathrm{w}}}} \frac{T_{1}\left(r_{b}\right)}{J_{1}\left(k_{\mathrm{n}} r_{b}\right)} .
\end{aligned}
$$

Note that $p_{\mathrm{n}}(r)$ and $p_{\mathrm{w}}(r)$ are also frequency-dependent, as $k_{\mathrm{n}}$ and $k_{\mathrm{w}}$ are given by

$$
\begin{aligned}
& k_{\mathrm{w}}(\omega)=\left(\frac{12 \eta_{\mathrm{w}}}{i h_{0}^{2} K_{\mathrm{w}}} \omega\right)^{\frac{1}{2}}, \\
& k_{\mathrm{n}}(\omega)=\left(\frac{12 \eta_{\mathrm{n}}}{i h_{0}^{2} K_{\mathrm{n}}} \omega\right)^{\frac{1}{2}} .
\end{aligned}
$$

A detailed derivation of Eqs (17) to (22) is given in Appendix A. 
We now return to the problem of obtaining the dynamic bulk modulus of the partially-saturated crack $K^{*}$ (Eq. 1). The first term of Eq. (2) is the force exerted by the non-wetting fluid phase which, using Eq. (18), responds to

$$
\begin{aligned}
\Delta F_{\mathrm{n}} & =2 \pi \int_{0}^{r_{b}} p_{\mathrm{n}}(r) r \mathrm{~d} r, \\
& =-\pi r_{c}^{2} S_{\mathrm{n}} \mathcal{T}_{\mathrm{n}}(\omega) \frac{\Delta h K_{\mathrm{n}}}{h_{0}},
\end{aligned}
$$

where $\mathcal{T}_{\mathrm{n}}(\omega)$ is given by

$$
\mathcal{T}_{\mathrm{n}}(\omega)=\left(1-A_{\mathrm{n}}^{*} \frac{2 J_{1}\left(k_{\mathrm{n}} r_{b}\right)}{k_{\mathrm{n}} r_{b} K_{\mathrm{n}}}\right) .
$$

On the other hand, the second term of Eq. (2) is the force exerted by the wetting fluid phase which, using Eq. (17), responds to

$$
\begin{aligned}
\Delta F_{\mathrm{w}} & =2 \pi \int_{r_{b}}^{r_{c}} p_{\mathrm{w}}(r) r \mathrm{~d} r, \\
& =-\pi r_{c}^{2} S_{\mathrm{w}} \mathcal{T}_{\mathrm{w}}(\omega) \frac{\Delta h K_{\mathrm{w}}}{h_{0}},
\end{aligned}
$$

where $\mathcal{T}_{\mathrm{w}}(\omega)$ is given by

$$
\mathcal{T}_{\mathrm{w}}(\omega)=\left[\frac{1}{S_{\mathrm{w}}}-\frac{S_{\mathrm{n}}}{S_{\mathrm{w}}}\left(1-\mathcal{B}_{\mathrm{w}}^{*} \frac{2 T_{1}\left(r_{b}\right)}{r_{b} k_{\mathrm{w}} K_{\mathrm{w}}}\right)\right]
$$

Again, a detailed derivation of Eqs (25) to (28) is given in Appendix A.

The expression for the effective crack stiffness (Eq. 1) is obtained by considering Eqs (25) and (27), that is,

$$
K^{*}=\pi r_{c}^{2}\left[S_{\mathrm{w}} K_{\mathrm{w}} \mathcal{T}_{\mathrm{w}}(\omega)+S_{\mathrm{n}} K_{\mathrm{n}} \mathcal{T}_{\mathrm{n}}(\omega)\right] \frac{1}{h_{0}} .
$$

The functions $\mathcal{T}_{\mathrm{w}}(\omega)$ and $\mathcal{T}_{\mathrm{w}}(\omega)$ present some key characteristics with frequency. On the one hand, both tend to unity for sufficiently high frequencies, that is, $\lim _{\omega \rightarrow \infty} \mathcal{T}_{\mathrm{n}}(\omega)=\lim _{\omega \rightarrow \infty} \mathcal{T}_{\mathrm{w}}(\omega)=1$. For sufficiently high frequencies, fluid pressure gradients arising between the wetting and the non-wetting fluid phases do not have enough time to equilibrate in a half-wave-cycle and, thus, the crack presents its highest stiffness and viscous flow and dissipation are negligible. In this context, the crack is in the unrelaxed state, and its stiffness converges to

$$
\lim _{\omega \rightarrow \infty} K^{*}(\omega)=\frac{\pi r_{c}^{2}}{h_{0}} K_{f, \infty}^{*}
$$

where the high-frequency effective bulk modulus is given by $K_{f, \infty}^{*}=\left[S_{\mathrm{w}} K_{\mathrm{w}}+S_{\mathrm{n}} K_{\mathrm{n}}\right]$, which is the Voigt (isostrain) average of the corresponding fluid bulk moduli (Mavko et al. 2009).

On the other hand, for sufficiently small frequencies, the crack is in a relaxed state, that is, the pressure gradients have time to relax in a half-wave-cycle. In this regime, viscous dissipation is virtu- 
ally null and the medium presents its lowest stiffness. This low-frequency limit is given by

$$
\begin{aligned}
& \lim _{\omega \rightarrow 0} \mathcal{T}_{\mathrm{w}}(\omega)=\left[S_{\mathrm{w}}+\frac{K_{\mathrm{w}}}{K_{\mathrm{n}}} S_{\mathrm{n}}\right]^{-1}, \\
& \lim _{\omega \rightarrow 0} \mathcal{T}_{\mathrm{n}}(\omega)=\frac{K_{\mathrm{w}}}{K_{\mathrm{n}}}\left[S_{\mathrm{w}}+\frac{K_{\mathrm{w}}}{K_{\mathrm{n}}} S_{\mathrm{n}}\right]^{-1},
\end{aligned}
$$

and, thus,

$$
\lim _{\omega \rightarrow 0} K^{*}(\omega)=\frac{\pi r_{c}^{2}}{h_{0}} K_{f, 0}^{*}
$$

with $K_{f, 0}^{*}=\left[\frac{S_{\mathrm{w}}}{K_{\mathrm{w}}}+\frac{S_{\mathrm{n}}}{K_{\mathrm{n}}}\right]^{-1}$, which is the Reuss (isostress) average of the fluid bulk moduli.

For intermediate frequencies, pressure gradients arising within the partially saturated crack produce viscous dissipation due to squirt flow, which, in turn, affects amplitudes and velocities of traveling seismic waves. A comparison of Eqs (29), (30), and (33) shows that, at any given frequency, the corresponding crack stiffness can be obtained by conceptualising the crack as saturated with a modified fluid whose effective bulk modulus responds to

$$
K_{f}^{*}(\omega)=S_{\mathrm{w}} K_{\mathrm{w}} \mathcal{T}_{\mathrm{w}}(\omega)+S_{\mathrm{n}} K_{\mathrm{n}} \mathcal{T}_{\mathrm{n}}(\omega)
$$

Eq. (34) is the central methodological result of this paper. As shown below, considering this effective complex-valued and frequency-dependent fluid bulk modulus in Hudson's (1981) model permits squirt flow effects to be included in the corresponding analytical solution.

\subsection{Effective compliance matrix of the medium}

Hudson $(1980,1981)$ derived simple expressions for the components of the effective compliance matrix $\mathbf{c}^{\text {eff }}$ of an elastic solid comprising aligned thin penny-shaped cracks or inclusions, with normals aligned along the vertical axis $z$. The first order solution considers that

$$
\mathbf{c}^{\mathrm{eff}}=\mathbf{c}^{0}+\varepsilon \mathbf{c}^{1},
$$

where $\mathbf{c}^{0}$ denotes the compliance matrix of the isotropic background, $\mathbf{c}^{1}$ the first-order excess compliance of the rock due to the presence of the fractures, and $\varepsilon=\nu r_{c}^{3}$ denotes the crack density, with $r_{c}$ being the radius of the penny shaped cracks and $\nu=N / V$ the number of cracks $N$ per unit volume $V$. Recall that Hudson's model assumes a low crack density, that is, $\varepsilon<0.1$ and, thus, interactions between neighbouring cracks are considered to be negligible.

Using Voigt's notation, the isotropic background moduli are given by

$$
\begin{aligned}
& c_{11}^{0}=c_{33}^{0}=\lambda_{s}+2 \mu_{s}, \\
& c_{13}^{0}=\lambda_{s}, \\
& c_{44}^{0}=\mu_{s},
\end{aligned}
$$


with $\lambda_{s}$ and $\mu_{s}$ Lamé's parameters of the solid background. The first-order corrections are given by

$$
\begin{aligned}
c_{11}^{1} & =-\frac{\lambda_{s}^{2}}{\mu_{s}} U_{3}, \\
c_{13}^{1} & =-\left(\lambda_{s}+2 \mu_{s}\right) \frac{\lambda_{s}}{\mu_{s}} U_{3}, \\
c_{33}^{1} & =-\left(\lambda_{s}+2 \mu_{s}\right)^{2} \frac{U_{3}}{\mu_{s}}, \\
c_{44}^{1} & =-\mu_{s} U_{1} . \\
c_{66}^{1} & =0
\end{aligned}
$$

As the medium is effectively VTI, the corrections for the remaining components respond to $c_{22}^{1}=c_{11}^{1}$, $c_{55}^{1}=c_{44}^{1}$, and $c_{i j}^{1}=c_{j i}^{1}$. Hudson $(1980,1981)$ gave the following expressions for fluid-filled cracks

$$
\begin{aligned}
U_{3} & =\frac{4\left(\lambda_{s}+2 \mu_{s}\right)}{3\left(\lambda_{s}+\mu_{s}\right)} \frac{1}{1+\mathcal{K}} \\
U_{1} & =\frac{16\left(\lambda_{s}+2 \mu_{s}\right)}{3\left(3 \lambda_{s}+4 \mu_{s}\right)}
\end{aligned}
$$

with

$$
\mathcal{K}=\frac{K_{f}\left(\lambda_{s}+2 \mu_{s}\right)}{\pi \alpha \mu_{s}\left(\lambda_{s}+\mu_{s}\right)}
$$

where $\alpha=c / 2 r_{c}$ denotes the crack aspect ratio, with $c=\frac{3}{2} h_{0}$ being the ellipsoidal mean aperture.

In this work, we propose to consider $K_{f}^{*}$ (Eq. 34) as the fluid bulk modulus in Eq. (46), rendering the saturated effective compliance matrix saturation- and frequency-dependent. Even though not shown here, as it is a more complicated approach, one could consider a dry frame, $K_{f}=0$ in Eq. (46), and then use Gassmann's equations (Appendix B) to saturate the medium with the effective fluid $K_{f}^{*}$ and obtain virtually identical results. Also, it is important to remark that Hudson's (1980; 1981) model is chosen here due to the simplicity of the corresponding equations. However, alternative models, such as, the Eschelby-Cheng equations (Cheng 1993) provide similar results when saturated with $K_{f}^{*}$. In this sense, Hudson's model for arbitrary crack orientations can be employed to analyse squirt flow effects in isotropic media containing partially saturated cracks (Hudson 1981; Pointer et al. 2000).

\subsection{Phase velocities}

For the above described VTI medium, the solutions of the wave equation permit one to compute the phase velocities associated with the quasi-compressional $\hat{V}_{p}$, quasi-shear $\hat{V}_{s}$ modes, which are given by (e.g., Mavko et al. 2009)

$$
\begin{aligned}
\hat{V}_{p}(\theta) & =\left[c_{11}^{\mathrm{eff}}(\sin \theta)^{2}+c_{33}^{\mathrm{eff}}(\cos \theta)^{2}+c_{44}^{\mathrm{eff}}+\sqrt{\mathcal{M}}\right]^{\frac{1}{2}}\left(2 \rho_{b}\right)^{-\frac{1}{2}}, \\
\hat{V}_{s v}(\theta) & =\left[c_{11}^{\mathrm{eff}}(\sin \theta)^{2}+c_{33}^{\mathrm{eff}}(\cos \theta)^{2}+c_{44}^{\mathrm{eff}}-\sqrt{\mathcal{M}}\right]^{\frac{1}{2}}\left(2 \rho_{b}\right)^{-\frac{1}{2}},
\end{aligned}
$$


Table 1. Material properties of the cracked rock models employed in this study. Solid grain properties of media 1 and 2 correspond to those of a Westerly granite (Hadley 1976) and a Poisson solid (Cheng 1993), respectively.

\section{Solid Phase $\quad$ Medium 1 Medium 2}

\begin{tabular}{lccc}
\hline$K_{s}[\mathrm{GPa}]$ & 56 & 65 & \\
$\mu_{s}[\mathrm{GPa}]$ & 33 & 39 & \\
$\alpha$ & $3.8 \times 10^{-3}$ & 0.01 & \\
$\epsilon$ & $4.6 \times 10^{-3}$ & 0.12 & \\
$h_{0}[\mu \mathrm{m}]$ & 10 & 100 & \\
\hline Fluid Phase & Glycerin & Air & Water \\
\hline$K_{f}[\mathrm{GPa}]$ & 4.35 & $1 . \times 10^{-4}$ & 2.2 \\
$\eta_{f}[\mathrm{~Pa} . \mathrm{s}]$ & 1 & $2 . \times 10^{-5}$ & 0.001 \\
\hline
\end{tabular}

with $\theta$ denoting the angle between the wave vector and the $z$-axis of symmetry (i.e., $\theta=0^{\circ}$ for propagation along the $z$-axis) and $\rho_{b}=\rho_{s}(1-\phi)+\phi\left(S_{\mathrm{w}} \rho_{\mathrm{w}}+S_{\mathrm{n}} \rho_{\mathrm{n}}\right)$ the bulk density, with $\rho_{s}$ denoting the density of the solid background. The parameter $\mathcal{M}$ responds to (e.g., Mavko et al. 2009)

$$
\mathcal{M}=\left[\left(c_{11}^{\mathrm{eff}}-c_{44}^{\mathrm{eff}}\right)(\sin \theta)^{2}-\left(c_{33}^{\mathrm{eff}}-c_{44}^{\mathrm{eff}}\right)(\cos \theta)^{2}\right]^{2}+\left(c_{13}^{\mathrm{eff}}-c_{44}^{\mathrm{eff}}\right)^{2}(\sin 2 \theta)^{2}
$$

If the effective compliance matrix of the medium is computed using $K_{f}^{*}\left(S_{\mathrm{w}}, \omega\right)$ (Eq. 34) as saturating fluid, the velocities $\hat{V}_{j}$, with $j=p, s v$, become complex-valued and frequency-dependent. The corresponding equivalent phase velocities and inverse quality factors are given by (e.g., Rubino et al. 2016)

$$
V_{j}\left(\theta, \omega, S_{\mathrm{w}}\right)=\left[\Re\left\{\frac{1}{\hat{V}_{j}\left(\theta, \omega, S_{\mathrm{w}}\right)}\right\}\right]^{-1}, \quad Q_{j}^{-1}\left(\theta, \omega, S_{\mathrm{w}}\right)=\frac{\Im\left\{\hat{V}_{j}\left(\theta, \omega, S_{\mathrm{w}}\right)^{2}\right\}}{\Re\left\{\hat{V}_{j}\left(\theta, \omega, S_{\mathrm{w}}\right)^{2}\right\}},
$$

where $\Re$ and $\Im$ denote the real and imaginary parts, respectively. Velocity and attenuation of the pure shear $(\mathrm{SH})$ wave are not considered in this work because they are not affected by squirt flow process occurring in the considered VTI medium. 


\section{RESULTS}

In this section, we study the effects of squirt flow due to partial crack saturation on seismic phase velocities and amplitudes. The velocity dispersion and attenuation of $\mathrm{P}$-waves travelling in the vertical direction $\left(\theta=0^{\circ}\right)$, i.e., perpendicular to the cracks, are much larger than along other directions. We thus first focus the analysis on this particular scenario. Then, we explore the corresponding effects on the full stiffness matrix, which is used to calculate the seismic properties for all incidence angles and wave modes.

\subsection{Crack saturation effects on P-wave modulus dispersion and attenuation for normal incidence}

Here, we focus our analysis on the effects of squirt flow due to partially saturated cracks on P-waves for normal incidence. For this, we consider an elastic medium with the properties of a Westerly granite (e.g., Hadley 1976) that contains aligned cracks. Cracks are partially saturated with glycerin (wetting fluid) and air (non-wetting fluid), as such fluid phases are commonly used to study squirt flow effects in laboratory settings (e.g., Batzle et al. 1996; Fortin et al. 2014). The properties of the solid, fluids, and cracks are summarised in Table 1. We employ Eq. (34) to obtain bulk modulus of the effective fluid $K_{f}^{*}\left(\omega, S_{\mathrm{w}}\right)$. Then, we make use of Hudson's model (Section 2.5), considering $K_{f}^{*}\left(\omega, S_{\mathrm{w}}\right)$ in Eq. (46) as the fluid bulk modulus. When computing the functions $\mathcal{T}_{\mathrm{n}}$ (Eq. 26) and $\mathcal{T}_{\mathrm{w}}$ (Eq. 28) from the parameters given in Table 1, we take

$$
\begin{aligned}
& r_{c}=3 h_{0} / 4 \alpha, \\
& r_{b}=r_{c} \sqrt{1-S_{\mathrm{w}}} .
\end{aligned}
$$

It is important to remark that squirt flow effects are controlled by the behaviour of the effective fluid bulk modulus $K_{f}^{*}\left(S_{\mathrm{w}}, \omega\right)=S_{\mathrm{w}} K_{\mathrm{w}} \mathcal{T}_{\mathrm{w}}(\omega)+\left(1-S_{\mathrm{w}}\right) K_{\mathrm{n}} \mathcal{T}_{\mathrm{n}}(\omega)$ (Equation 34). Let us analyse first the behaviour of $\mathcal{T}_{\mathrm{n}}, \mathcal{T}_{\mathrm{w}}$, and $K_{f}^{*}$ as functions of frequency. We observe that, for $S_{\mathrm{w}}=0.99, \mathcal{T}_{\mathrm{n}}$ decreases with frequency, while $\mathcal{T}_{\mathrm{w}}$ values increase (Fig. 2a). Interestingly, $\mathcal{T}_{\mathrm{n}}$ and $\mathcal{T}_{\mathrm{w}}$ exhibit different inflection points in the frequency domain. As expected, the absolute value of $K_{f}^{*}$ as a function of frequency is bounded by the isostrain and isostress limits (Fig. 2b). We also analyzed the effects of surface tension, by considering $\sigma=0 \mathrm{mN} / \mathrm{m}$ and $\sigma=63 \mathrm{mN} / \mathrm{m}$, the latter being the approximate surface tension of air-glycerine interfaces at $20^{\circ} \mathrm{C}$, for which $\gamma \simeq 0^{\circ}$ (e.g., Takamura et al. 2012). We note that the effective fluid bulk modulus remains virtually unperturbed by these variations. We have analysed this for the full range of saturations and a great variety of crack aspect ratios and, indeed, the effects of capillary forces can be regarded as negligible for the considered model (Fig. 2b). This characteristic will be further discussed in Section 4. 

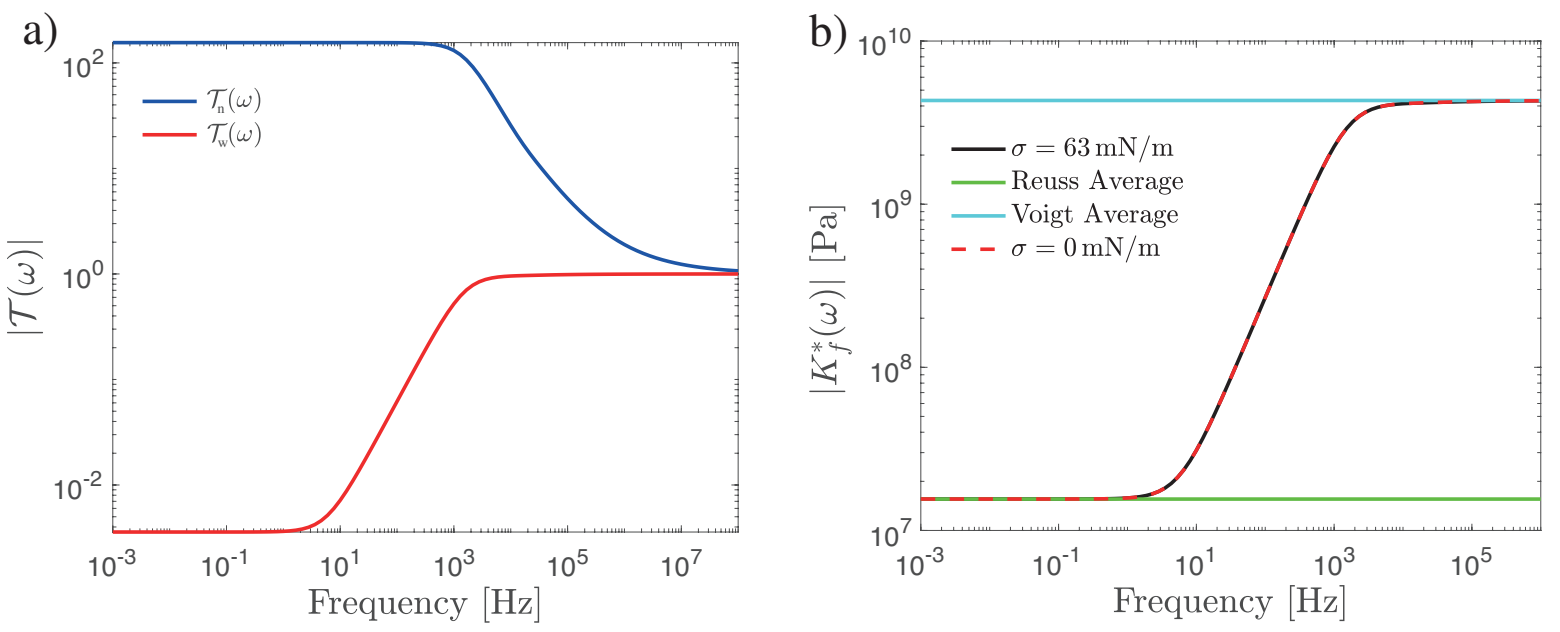

Figure 2. (a) Absolute values of $\mathcal{T}_{\mathrm{n}}$ (blue line), $\mathcal{T}_{\mathrm{w}}$ (red line), and of the (b) effective fluid bulk modulus $K_{f}^{*}$ as functions of frequency. We consider a crack of aperture $h_{0}=10 \mu \mathrm{m}$, aspect ratio $\alpha=3.8 \times 10^{-3}$, and saturated with glycerin $\left(S_{\mathrm{w}}=0.99\right)$ and air. In panel (b), we illustrate the low-frequency (green line) and high-frequency (light blue line) limits and, also, solutions considering (black line) and disregarding (dashed red line) capillary tension effects $\sigma$ at the interface between the immiscible fluid phases.

Squirt flow occurring between the stiff wetting fluid phase and the more compliant non-wetting phase induces a dispersive behavior of the plane wave modulus $\Re\left\{c_{33}\right\}$ (Fig. 3a). We note that decreasing the wetting phase saturation $S_{\mathrm{w}}$, which entails augmenting the radius $r_{b}$ (see Fig. 1), shifts the dispersion curves towards higher frequencies (Fig. 3a). In the low-frequency limit, the effective bulk modulus of the fluid responds to the harmonic mean of the corresponding fluid bulk moduli. In this context, cracks behave as air-saturated, unless $S_{\mathrm{w}}$ approaches unity, due to the fact that air has a much smaller bulk moduli than glycerin. This is indeed what governs $\Re\left\{c_{33}\right\}$ in the low-frequency limit, where values remain virtually unchanged for $S_{\mathrm{w}} \leq 0.9$ (Fig. 3a), and increase when $S_{\mathrm{w}}$ approaches unity (Fig. 3a, light blue line). Attenuation curves, on the other hand, are characterised by a peak value that slightly decreases and moves towards higher frequencies with decreasing saturation (Fig. 3b). Interestingly, by relating the frequencies associated with the attenuation peaks $f_{c}$ (Fig $3 b$ ) with the corresponding $S_{\mathrm{w}}$ values, we observe that $f_{c} \propto 1 / S_{\mathrm{w}}^{3}$. During compression of the cracks, the relatively more compliant non-wetting phase is invaded by the stiffer wetting phase. For decreasing $S_{\mathrm{w}}$, the central non-wetting phase bubble grows, increasing the contact surface between both immiscible phases which, in turn, implies that the wetting fluid has a larger area for flow and, thus, pressure relaxation occurs faster, i.e. at higher frequencies. We remark that the aspect ratio, the viscosity of the fluids, and $K_{s}$ also play an important role in determining $f_{c}$ in squirt flow processes (e.g., Gurevich et al. 2010). Also, is interesting to note that attenuation levels are significant for all considered saturations (Fig. 3b). This characteristic shows that squirt flow in partially saturated cracks does not 

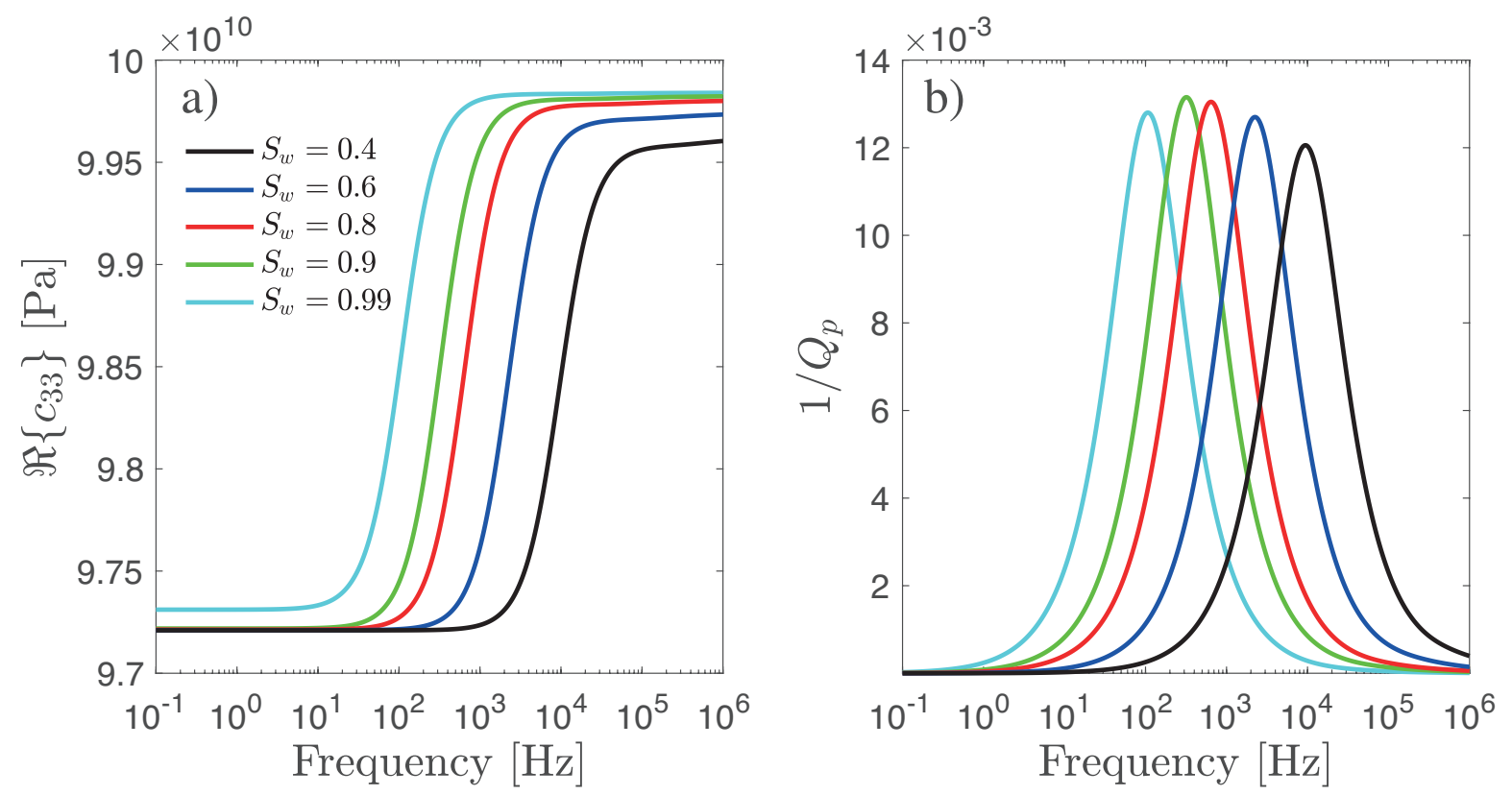

Figure 3. (a) Plane wave modulus $\Re\left\{c_{33}\right\}$ and (b) inverse quality factor $Q_{p}^{-1}$ as functions of frequency for vertically travelling P-waves in a solid background containing a regular distribution of horizontal partially saturated cracks. We illustrate the corresponding behavior for different for different saturations (coloured lines). Rock and fluid properties correspond to those of Westerly granite, that is, medium 1, saturated with glycerin and air (Table 1).

follow the general behavior of the so-called patchy saturation models in porous media (e.g., White 1975; White et al. 1975; Johnson 2001), where attenuation becomes only significant at relatively high saturations $\left(S_{\mathrm{w}}>0.8\right)$, but tend to assume considerably small values for lower saturation levels.

The frequency- and saturation-dependent behavior illustrated in Fig. 3 can also be analysed for fixed frequencies and varying saturation (Fig. 4). As expected, we observe that the plane wave modulus increases with frequency and saturation (Fig. 4a). $\Re\left\{c_{33}\right\}$ values are bounded by Reuss and Voigt pore fluid stiffness approximations for the saturating fluid (Fig. 4a). Attenuation estimates as functions of saturation present peak values located at smaller saturations for increasing frequencies (Fig. 4b). This is a highly interesting feature that, again, does not follow the behavior of mesoscopic patchy saturation models (Yin et al. 1992; Cadoret et al. 1998; Rubino \& Holliger 2012; Solazzi et al. 2017, 2019), where attenuation peaks are located at $S_{\mathrm{w}} \geq 0.8$ irrespective of the probing frequency.

\subsection{Effects of partial saturation on the anisotropy of $V_{p}$ and $V_{s}$}

The presence of aligned cracks can lead to substantial seismic anisotropy. Squirt flow occurring within partially saturated cracks may render this anisotropic response frequency-dependent. In the following, we explore the behavior of $V_{p}$ and $V_{s v}$ as functions of the incidence angle $\vartheta$ for different frequencies 

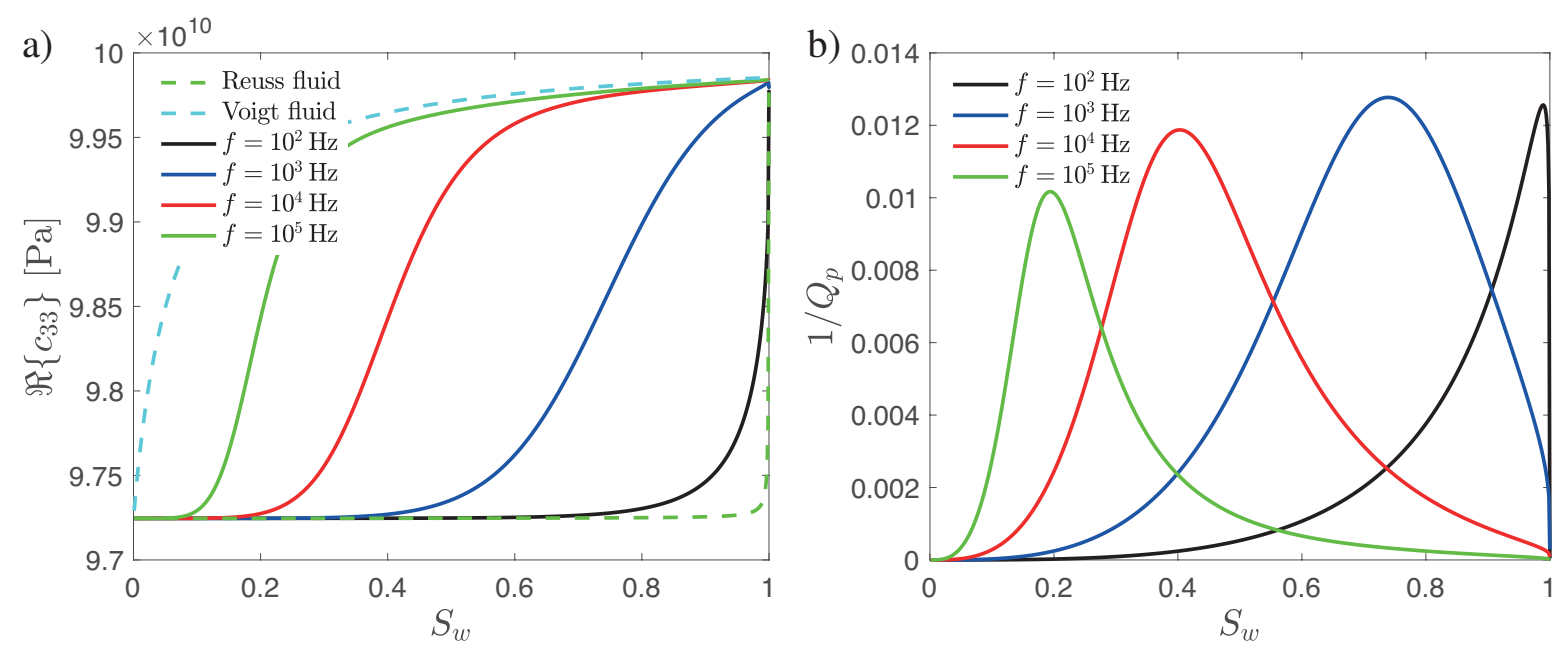

Figure 4. (a) Plane wave modulus $\Re\left\{c_{33}\right\}$ and (b) inverse quality factor $Q_{p}^{-1}$ as functions of saturation for vertically travelling P-waves in a solid background containing a regular distribution of horizontal partially saturated cracks. We illustrate the corresponding behavior for different frequencies (coloured lines). Rock and fluid properties correspond to those of Fig. 3. In panel (a), we also plot the response of the partially saturated medium when the effective fluid responds to Reuss (dashed green line) and Voigt (dashed light blue line) end-member behaviour.

and saturations (Fig. 5). In the analysis, we take the properties proposed by Cheng (1993), that is, we consider a Poisson solid (i.e., $\lambda_{s}=\mu_{s}$ ), whose properties are summarised in Table 1 . The saturating fluids are water and air, with densities $\rho_{\mathrm{w}}=1000 \mathrm{~kg} / \mathrm{m}^{3}$ and $\rho_{\mathrm{n}}=1 \mathrm{~kg} / \mathrm{m}^{3}$, respectively. We consider that the density of the medium is $\rho_{s}=2640 \mathrm{~kg} / \mathrm{m}^{3}$ and its porosity $\phi=\epsilon \pi h_{0} / r_{c}$. We compare the results of our model with those predicted by Hudson's theory for dry and fully-saturated cracks, which act as end-member anisotropic responses of the medium. Recall that squirt flow effects are expected to be maximal for P-waves at $\vartheta=0^{\circ}$ and for S-waves at $\vartheta=45^{\circ}$. This characteristic is associated with the capability of the corresponding wave modes to induce larger fluid pressure gradients within the cracks for such directions of propagation.

Let us analyse the anisotropic response of $V_{p}$ at a constant saturation $\left(S_{\mathrm{w}}=0.999\right)$ for probing frequencies of $1 \mathrm{kHz}$ and $1 \mathrm{MHz}$ (Fig. 5). We note that $V_{p}$ increases with incidence angle and varies with frequency (compare red and blue solid lines in Fig. 5a). As expected, velocity dispersion decreases with increasing $\vartheta$-values (observe the separation between red and blue solid lines in Fig. 5a). We note that even if the saturation of the medium remains constant, the anisotropic behavior of $V_{s v}$ is highly sensitive to squirt flow effects (compare red and blue dashed lines in Fig. 5a) and that the corresponding dispersion is maximal at intermediate angles. Conversely, for a constant frequency (1 MHz), varying the fluid content within cracks results in effective responses that shift between the dry and fully-saturated estimates predicted by Hudson's model (Fig. 5b). These results illustrate that 
a)

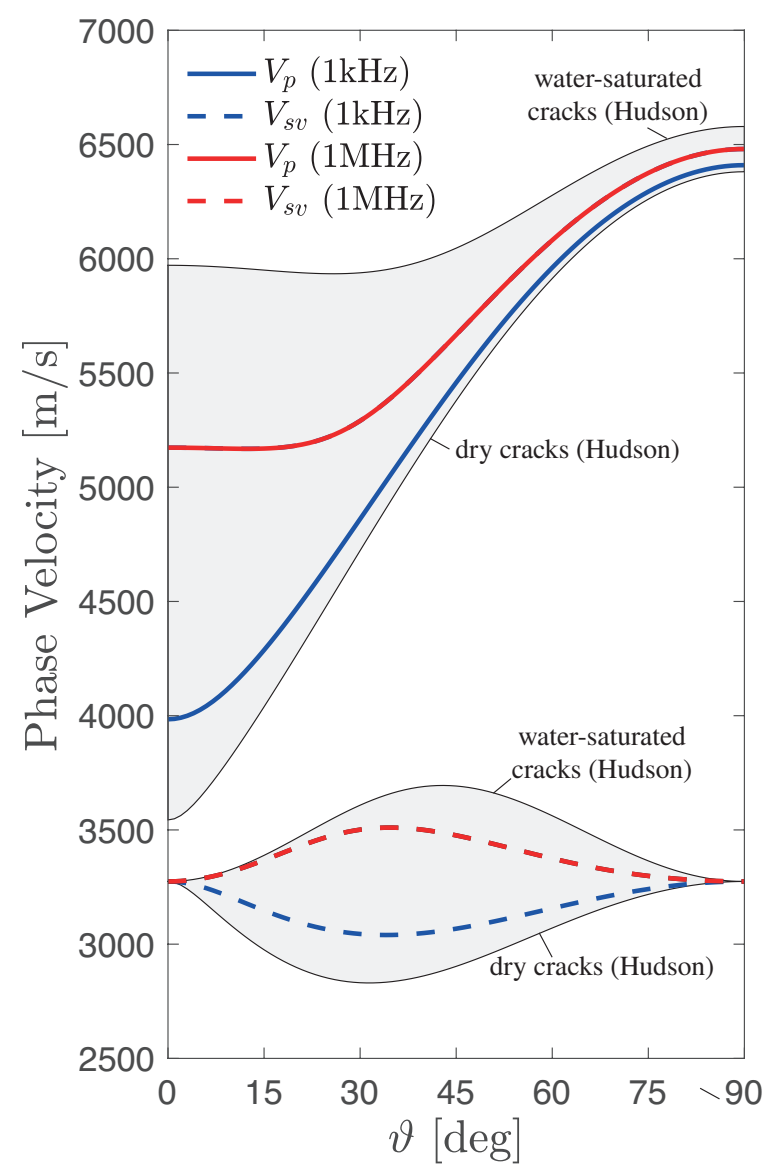

b)

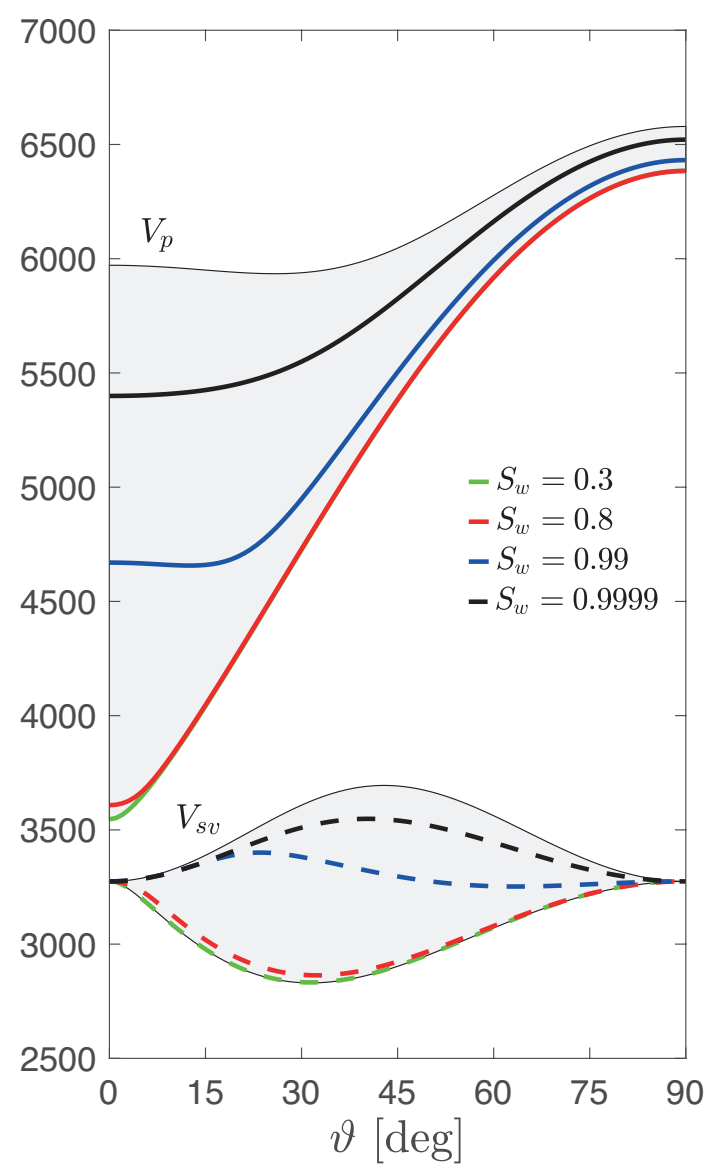

Figure 5. Phase velocities $V_{p}$ (solid lines) and $V_{s v}$ (dashed lines) as functions of the incidence angle $\vartheta$ for medium 2 (Table 1) saturated with water and air. In panel (a) we illustrate the results considering a fixed saturation of $S_{\mathrm{w}}=0.999$ and frequencies of $1 \mathrm{kHz}$ (blue lines) and $1 \mathrm{MHz}$ (red lines). In panel (b) we consider a fixed frequency of $1 \mathrm{MHz}$ and different saturations (coloured lines). Grey coloured regions in panels (a) and (b) highlight the zones delimited by the dry and fully-saturated anisotropic responses computed using Hudson's model.

squirt flow occurring within partially saturated cracks is an important phenomenon to contemplate when interpreting seismic data for fluid content estimation.

\section{DISCUSSION}

\subsection{Comparison with existing models}

As mentioned previously, alternative approaches can be followed to estimate the effects of squirt flow in partially saturated cracks. Previous analytical solutions present limitations, such as, Pointer et al. (2000) model (Appendix C), which is valid only at relatively low frequencies, leads to attenuation 
estimates that are linear with frequency, and predicts a null phase velocity dispersion. For their part, computational approaches, such as, finite element procedures, permit to simulate squirt flow within partially saturated cracks using suitable upscaling procedures. For instance, Quintal et al. $(2016,2019)$ proposed a finite element method to solve the coupled, linearised, and quasi-static Lamé-Navier and Navier-Stokes equations in a representative elementary volume (REV) of the medium of interest. This procedure allows to obtain the attenuation and phase velocity dispersion due to squirt flow occurring within a medium composed by an elastic background and a fluid filled pore space of defined geometry. Let us compare the analytical solution proposed in this study with this numerical approach as well as with the analytical model proposed by Pointer et al. (2000). For this, we consider a medium with the properties of a cracked Westerly granite (Table 1) where cracks are saturated with glycerin and air. The REV of this medium, considered in the finite element numerical solution, consists of a $12 \mathrm{~mm}$ side-length sample comprising a single crack characterised by $r_{c}=2.0 \mathrm{~mm}, r_{b}=0.16 \mathrm{~mm}$, and $h=0.01 \mathrm{~mm}$.

The attenuation derived from the proposed analytical model fits perfectly the low-frequency analytical prediction of Pointer et al. (2000) and agrees with the results obtained through the finite element 3D numerical approach of Quintal et al. (2019) (Fig. 6a). The numerical approach results in higher attenuation values than our analytical solution at high frequencies (compare blue circles and solid red line in Fig. 6a for $f>10^{4} \mathrm{~Hz}$ ). Similar results were observed by Alkhimenkov et al. (2020), who compared Gurevich's (2010) analytical solution with the same finite element numerical approach for mono-saturated cracks connected to toroidal pores. The reason for this discrepancy lies in the fact that our analytical model considers an homogeneous oscillatory strain imposed on the crack walls whereas, in the 3D finite element procedure, the strain field is applied at the boundaries of a REV of an elastic medium comprising a partially saturated crack. The differences in the result is associated with discrepancies in the strain along the crack walls, which do not deform in the radial direction in the proposed analytical model but may do so in the 3D numerical simulation. With respect to plane wave modulus dispersion, results (Fig. 6b) also show that the proposed model follows the general behavior of Pointer et al. (2000) model at low frequencies and those of the numerical simulations for the whole frequency range. Convergence with Gassmann-Wood (green solid line in Fig. 6b) and Gassmann-Hill (light blue solid line in Fig. 6b) limits, computed using Hudson's (1981) dry frame moduli and the anisotropic Gassmann equations (Appendix B), at low and high frequencies demonstrates that the analytical solution is well behaved. 

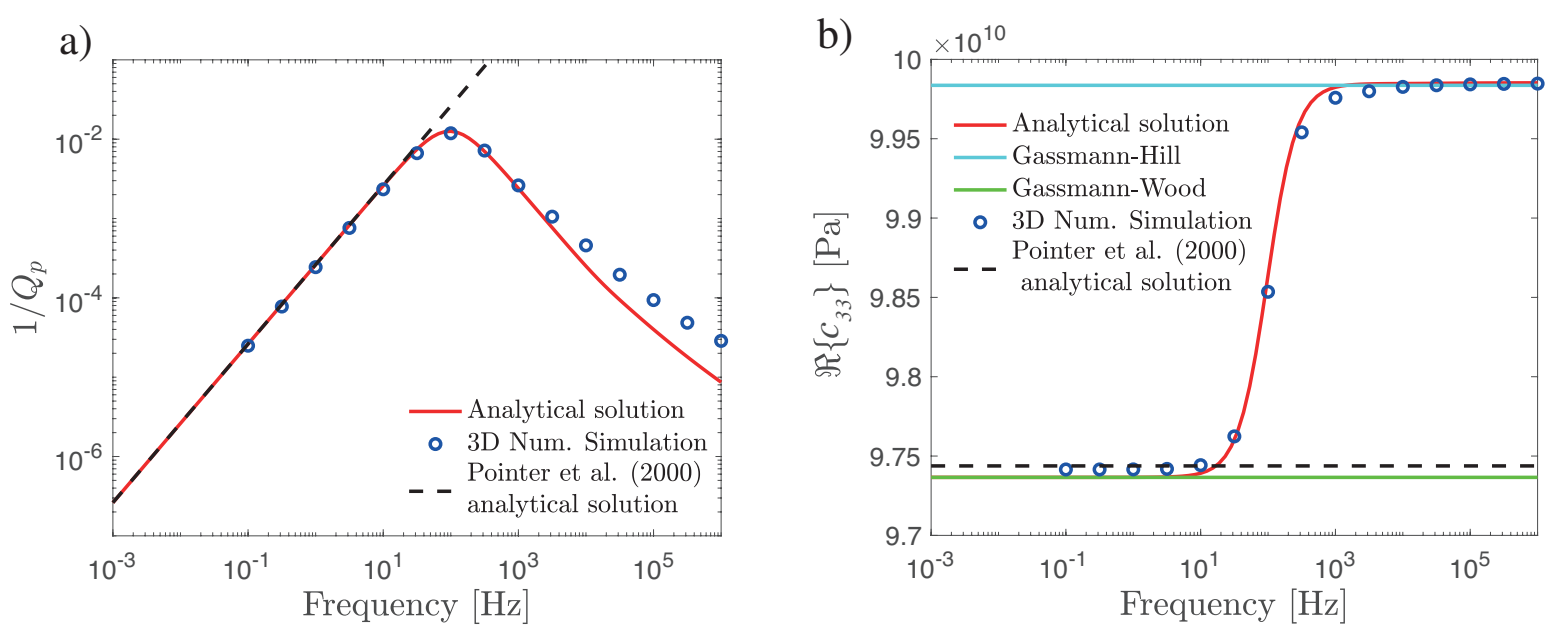

Figure 6. (a) Inverse quality factor $Q_{p}^{-1}$ and (b) modulus dispersion $\Re\left\{c_{33}\right\}$ as functions of frequency for vertically travelling P-waves in a solid background containing a regular distribution of horizontal partially saturated cracks. We illustrate the results provided by: (i) the proposed analytical solution (red solid line); (ii) the 3D numerical simulation (blue circles); and (iii) Pointer et al. (2000) low-frequency model. For completeness, in panel (b) we also illustrate the Gassmann-Hill (light blue line) and Gassmann-Wood (green line) limits.

\subsection{Outlook}

The proposed analytical solution also allows one to explore different aspects of the seismic properties of generic partially saturated rocks. For instance, Papageorgiou et al. (2016) discussed the effects of partial saturation on porous media to provide with a theoretical basis for the Brie et al. (1995) effective fluid model. In this context, Papageorgiou et al. (2016) argue that the effective fluid bulk modulus of a partially saturated porous medium can be regarded as a real-valued and frequency-independent parameter $\hat{K}_{f}$ that responds to

$$
\hat{K}_{f}=S_{\mathrm{w}} K_{\mathrm{w}} \hat{T}_{\mathrm{w}}+\left(1-S_{\mathrm{w}}\right) K_{\mathrm{n}} \hat{T}_{\mathrm{n}}
$$

with

$$
\begin{aligned}
& \hat{T}_{\mathrm{w}}=\left[S_{\mathrm{w}}+\hat{\alpha}\left(1-S_{\mathrm{w}}\right)\right]^{-1}, \\
& \hat{T}_{\mathrm{n}}=\hat{\alpha}\left[S_{\mathrm{w}}+\hat{\alpha}\left(1-S_{\mathrm{w}}\right)\right]^{-1},
\end{aligned}
$$

where $1 \leq \hat{\alpha} \leq K_{\mathrm{w}} / K_{\mathrm{n}}$ is a parameter related to the capillary pressure and depends on the specific fluid distribution within the pores (Papageorgiou et al. 2016). Eq. (53) shows remarkable similarities with Eq. (34), thus showing a potential relationship between $\mathcal{T}_{\mathrm{n}}$ and $\mathcal{T}_{\mathrm{w}}$ (Eqs 26 and 28), and $\hat{\alpha}$. This similarity points to the need of further analysis considering more complex crack/pore geometries, as both the fluid distribution (determined by capillary forces) and local squirt flow processes should certainly play a role in determining the effective fluid properties. 
It is also interesting to note that, in porous rocks having stiff large pores connected to compliant flat cracks, such as, the geometry proposed by Murphy et al. (1986), the non-wetting phase is expected to preferentially invade larger pores. In such a case, the squirt flow mechanism analyzed in this work will have negligible effects, as cracks would hardly be partially saturated. However, in rocks having only compliant porosity (e.g., Sarout et al. 2017), the non-wetting phase will locate at the maximum apertures of the cracks or compliant pores. Although, in this work we consider a simple case of cracks having constant aperture, squirt flow in partially saturated rough-walled cracks may indeed play a non-negligible role in a wide variety of pertinent scenarios. In particular, partially saturated squirt flow associated with residual non-wetting phase bubbles trapped in large pores/crack apertures could potentially lead to miss-interpretation of measurement in allegedly fully saturated media. In fact, the results in Fig. 5a suggest that, for $S_{\mathrm{w}}=0.999$, dispersion and attenuation can be significative even for nearly fully saturated rocks. The case of partially saturated and intersecting cracks it is certainly interesting, as squirt flow can also arise between cracks, and, in this context, the effects of partial saturation remain enigmatic.

We remark that the squirt flow model derived in this work (i) is limited to a low concentration of cracks, as it is an inherent assumption in Hudson (1981) model; (ii) disregards inertial effects associated with Biot's intrinsic dissipation, as we emulate the approach of Gurevich et al. (2010) to derive the effective crack stiffness; (iii) assumes that the seismic wavelengths are much larger than the crack sizes, in order to obtain the effective response of the medium. Some of these limitations and assumptions can be avoided. On the one hand, for large crack concentrations, self-consistent models may be employed to obtain the effective elastic properties accounting for crack interactions (e.g., Henyey \& Pomphrey 1982; Berryman et al. 2002). On the other hand, for seismic wavelengths comparable to the size of the cracks, scattering and squirt flow effects are expected to be coupled. In this sense, one could consider our analytical solution to obtain the frequency-dependent effective fluid bulk modulus and, then, use it for the saturating fluids, for instance, in the scattering solutions of 3D penny-shaped cracks proposed by Guo \& Gurevich (2020). Extensions of the proposed model to account for such effects are to be addressed in future works.

As previously stated, the proposed model is not sensitive to the capillary pressure at the interface between fluid phases and solid matrix. This behavior is related to the assumptions and geometries considered in our study. In this sense, we consider constant-aperture penny-shaped cracks in order to obtain an analytical solution. For cracks containing variable apertures, large strains could lead to irreversible modifications in the pore fluid distribution. Indeed this process was proposed by Beresnev et al. (2005) and Pride et al. (2008) as a possible fluid mobilisation mechanism using seismic waves. On the other hand, contact line slip and angle hysteresis conditions along the interface between fluids 
and the crack walls are not accounted for in the proposed model. These effects could, under certain conditions, play a key role in the flow characteristics (e.g., Miksis 1988; Andrew et al. 2014; Rozhko \& Bauer 2019) and, thus, further developments in this direction are certainly interesting. Finally, our model disregards the possible stiffening effects associated with capillary tension pulling on the crack walls. The latter effect is similar to that predicted by Bishop \& Blight (1963) in unconsolidated granular sediments and merits further research.

\section{CONCLUSIONS}

We have derived a new model to describe the effects of squirt flow in rocks containing aligned partially saturated penny-shaped cracks. The approach is based on solving the linearised Navier-Stokes equations within a partially saturated crack undergoing oscillatory variations in aperture that mimic the deformation caused by a passing seismic wavefield. For this, we consider the effects of the compressibility and viscosity of both immiscible pore fluid phases, which were usually disregarded in previous approaches. We derive the expression for an effective fluid, which describes the frequency-dependent stiffness variations of the partially saturated cracks which, when combined with well known analytical solutions, allows to retrieve the effective complex-valued and frequency-dependent compliance matrix of the VTI medium. Our model permits a straightforward interpretation of body wave phase velocities and attenuations as functions of the frequency and incidence angle in terms of the saturation state. The proposed analytical solution is validated by comparison with 3D numerical simulations and analytical models available in the literature. We show that the peak attenuation is shifted towards lower frequencies with increasing saturations. In contrast to mesoscopic patchy saturation, attenuation curves as functions of saturation for cracked partially saturated media may present maximum values at relatively low saturations. We also show that dispersion characteristics for P- and SV-waves can be significantly influenced by squirt flow processes occurring within partially saturated cracks. These results further indicate that squirt flow effects in partially saturated cracks do not follow the general behaviour of mesoscopic patchy saturation models, which only predict measurable $\mathrm{P}$-wave attenuations for saturations approaching unity. We conclude that the analytical solution proposed in this study allows for a better understanding squirt flow processes in partially saturated rocks and, thus, may permit a better interpretation of seismic data for fluid content estimation.

\section{DATA AVAILABILITY}

Experimental and observational data were neither used nor created for this research. Mathematical expressions to replicate the results of this article are available in the article. 


\section{ACKNOWLEDGMENTS}

This project has received funding from the European Union's HORIZON 2020 research and innovation programme under the Marie Sktodowska-Curie grant agreement No. 101007851. Part of this work was completed within the Swiss Competence Center for Energy Research-Supply of Electricity with support of Innosuisse. J. G. R. acknowledges the financial support received from the Agencia Nacional de Promoción Científica y Tecnológica of Argentina (PICT 2017- 2976).

\section{APPENDIX A: DETAILED SOLUTION TO THE DIFFERENTIAL PROBLEM}

In the following, we present a detailed derivation of the expressions of the four integration constants introduced in Eqs (9) and (10), that is, of $\mathcal{A}_{\mathrm{w}}, \mathcal{A}_{\mathrm{n}}, \mathcal{B}_{\mathrm{w}}$ and $\mathcal{B}_{\mathrm{n}}$. Then, we derive the expressions for the pore fluid pressures and of the effective crack stiffness.

\section{A1 Pore fluid pressures}

First, let us consider the no-flow condition at the edge of the crack (Eq. 11). Under this assumption, Eq. (10) reduces to

$$
-\mathcal{A}_{\mathrm{w}} k_{\mathrm{w}} J_{1}\left(k_{\mathrm{w}} r_{c}\right)-\mathcal{B}_{\mathrm{w}} k_{\mathrm{w}} Y_{1}\left(k_{\mathrm{w}} r_{c}\right)=0
$$

where we have employed that $J_{0}^{\prime}\left(k_{\mathrm{w}} r\right)=-k_{\mathrm{w}} J_{1}\left(k_{\mathrm{w}} r\right)$ and $Y_{0}^{\prime}\left(k_{\mathrm{w}} r\right)=-k_{\mathrm{w}} Y_{1}\left(k_{\mathrm{w}} r\right)$ (Abramowitz $\&$ Stegun 1965). Therefore, the value for the second constant is given by:

$$
\mathcal{A}_{\mathrm{w}}=-\mathcal{B}_{\mathrm{w}} \frac{Y_{1}\left(k_{\mathrm{w}} r_{c}\right)}{J_{1}\left(k_{\mathrm{w}} r_{c}\right)}
$$

The pressure of the wetting and non-wetting fluids thus respond to

$$
\begin{aligned}
& p_{\mathrm{n}}(r)=\mathcal{A}_{\mathrm{n}} J_{0}\left(k_{\mathrm{n}} r\right)+\frac{C_{\mathrm{n}}}{k_{\mathrm{n}}^{2}}, \\
& p_{\mathrm{w}}(r)=\mathcal{B}_{\mathrm{w}} T_{0}(r)+\frac{C_{\mathrm{w}}}{k_{\mathrm{w}}^{2}},
\end{aligned}
$$

with

$$
T_{0}(r)=\left(Y_{0}\left(k_{\mathrm{w}} r\right)-\frac{Y_{1}\left(k_{\mathrm{w}} r_{c}\right)}{J_{1}\left(k_{\mathrm{w}} r_{c}\right)} J_{0}\left(k_{\mathrm{w}} r\right)\right) .
$$

Now we consider the continuity condition given by Eq. (13). Taking into account Eqs (A.3) and (A.4), we obtain

with

$$
\left.\mathcal{A}_{\mathrm{n}} \frac{k_{\mathrm{n}}}{D_{0, \mathrm{n}}} J_{1}\left(k_{\mathrm{n}} r\right)\right|_{r=r_{b}}=\left.\mathcal{B}_{\mathrm{w}} \frac{k_{\mathrm{w}}}{D_{0, \mathrm{w}}} T_{1}(r)\right|_{r=r_{b}}
$$

$$
T_{1}(r)=\left(Y_{1}\left(k_{\mathrm{w}} r\right)-\frac{Y_{1}\left(k_{\mathrm{w}} r_{c}\right)}{J_{1}\left(k_{\mathrm{w}} r_{c}\right)} J_{1}\left(k_{\mathrm{w}} r\right)\right) .
$$


After some algebra, Eq. (A.6) yields

$$
\begin{aligned}
\mathcal{A}_{\mathrm{n}} & =\mathcal{B}_{\mathrm{w}} \frac{D_{0, \mathrm{n}}}{J_{1}\left(k_{\mathrm{n}} r_{b}\right) k_{\mathrm{n}}} \frac{k_{\mathrm{w}}}{D_{0, \mathrm{w}}} T_{1}\left(r_{b}\right), \\
& =\mathcal{B}_{\mathrm{w}} \sqrt{\frac{\eta_{\mathrm{n}} K_{\mathrm{n}}}{\eta_{\mathrm{w}} K_{\mathrm{w}}}} \frac{T_{1}\left(r_{b}\right)}{J_{1}\left(k_{\mathrm{n}} r_{b}\right)} .
\end{aligned}
$$

Finally, we consider the continuity of pressures at the interface (Eq. 16). For this purpose, we crossdiferentiate Eqs (A.3), (A.4), and (A.8):

$$
\mathcal{B}_{\mathrm{w}}=\left(T_{0}\left(r_{b}\right)-\frac{k_{\mathrm{w}}}{k_{\mathrm{n}}} \frac{D_{0, \mathrm{n}}}{D_{0, \mathrm{w}}} \frac{J_{0}\left(k_{\mathrm{n}} r_{b}\right)}{J_{1}\left(k_{\mathrm{n}} r_{b}\right)} T_{1}\left(r_{b}\right)\right)^{-1}\left(\frac{C_{\mathrm{n}}}{k_{\mathrm{n}}^{2}}-\frac{C_{\mathrm{w}}}{k_{\mathrm{w}}^{2}}-\frac{2 \sigma \cos \gamma}{h_{0}^{2}} \Delta h\right) .
$$

Replacing the expressions for $C_{j}$ and $D_{0, j}$, with $j=\mathrm{w}, \mathrm{n}$, in Eq. (A.9), we obtain

$$
\begin{aligned}
\mathcal{B}_{\mathrm{w}} & =\left(T_{0}\left(r_{b}\right)-\sqrt{\frac{\eta_{\mathrm{n}} K_{\mathrm{n}}}{\eta_{\mathrm{w}} K_{\mathrm{w}}}} \frac{J_{0}\left(k_{\mathrm{n}} r_{b}\right)}{J_{1}\left(k_{\mathrm{n}} r_{b}\right)} T_{1}\left(r_{b}\right)\right)^{-1}\left(K_{\mathrm{w}}-K_{\mathrm{n}}-\frac{2 \sigma \cos \gamma}{h_{0}}\right) \frac{\Delta h}{h_{0}}, \\
& =\mathcal{B}_{\mathrm{w}}^{*} \frac{\Delta h}{h_{0}}
\end{aligned}
$$

with $\mathcal{B}_{\mathrm{w}}^{*}=\left(T_{0}\left(r_{b}\right)-\sqrt{\frac{\eta_{\mathrm{n}} K_{\mathrm{n}}}{\eta_{\mathrm{w}} K_{\mathrm{w}}}} \frac{J_{0}\left(k_{\mathrm{n}} r_{b}\right)}{J_{1}\left(k_{\mathrm{n}} r_{b}\right)} T_{1}\left(r_{b}\right)\right)^{-1}\left(K_{\mathrm{w}}-K_{\mathrm{n}}-\frac{2 \sigma \cos \gamma}{h_{0}}\right)$. This is the value of the fourth, and last, constant of integration of the problem. Note that $\mathcal{A}_{\mathrm{n}}$ of Eq. (A.8) can be also expressed as $\mathcal{A}_{\mathrm{n}}=\mathcal{A}_{\mathrm{n}}^{*} \frac{\Delta h}{h_{0}}$, with $\mathcal{A}_{\mathrm{n}}^{*}=\mathcal{B}_{\mathrm{w}}^{*} \sqrt{\frac{\eta_{\mathrm{n}} K_{\mathrm{n}}}{\eta_{\mathrm{w}}}} \frac{T_{\mathrm{w}}\left(r_{b}\right)}{J_{1}\left(k_{\mathrm{n}} r_{b}\right)}$. In conclusion, the pressures for the wetting and nonwetting fluids are given by Eqs (A.3) and (A.4), respectively, where the parameters $\mathcal{A}_{\mathrm{n}}$ and $\mathcal{B}_{\mathrm{w}}$ are given by Eqs (A.8) and (A.10), respectively. These results are expressed in the main text in Eqs (17) to (22).

\section{A2 Effective crack stiffness}

Let us return to the problem of obtaining the dynamic bulk modulus of the partially saturated crack $K^{*}$ (Eq. 1). For this, we need to compute the force $\Delta F$ given by Eq. (2). The corresponding first term is the force exerted by the non-wetting fluid phase, whose pressure is given by Eq. (A.3):

$$
\begin{aligned}
\Delta F_{\mathrm{n}} & =2 \pi \int_{0}^{r_{b}} p_{\mathrm{n}}(r) r \mathrm{~d} r \\
& =2 \pi \int_{0}^{r_{b}}\left(A_{\mathrm{n}} J_{0}\left(k_{\mathrm{n}} r\right) r+\frac{C_{\mathrm{n}} r}{k_{\mathrm{n}}^{2}}\right) \mathrm{d} r \\
& =\left.\pi\left(A_{\mathrm{n}} \frac{2 J_{1}\left(k_{\mathrm{n}} r\right) r}{k_{\mathrm{n}}}+\frac{C_{\mathrm{n}} r^{2}}{k_{\mathrm{n}}^{2}}\right)\right|_{0} ^{r_{b}}, \\
& =-\pi r_{b}^{2}\left(1-A_{\mathrm{n}}^{*} \frac{2 J_{1}\left(k_{\mathrm{n}} r_{b}\right)}{k_{\mathrm{n}} r_{b} K_{\mathrm{n}}}\right) \frac{\Delta h K_{\mathrm{n}}}{h_{0}} \\
& =-\pi r_{b}^{2} \mathcal{T}_{\mathrm{n}}(\omega) \frac{\Delta h K_{\mathrm{n}}}{h_{0}} \\
& =-\pi r_{c}^{2} S_{\mathrm{n}} \mathcal{T}_{\mathrm{n}}(\omega) \frac{\Delta h K_{\mathrm{n}}}{h_{0}}
\end{aligned}
$$


where we have used that $\left(\frac{\mathrm{d}}{\mathrm{d} z}\right)\left\{\frac{z}{a} J_{1}(a z)\right\}=z J_{0}(a z)$ (Abramowitz \& Stegun 1965). Also, we have employed that $r_{b}^{2}=r_{c}^{2}\left(1-S_{\mathrm{w}}\right)$, and defined $\mathcal{T}_{\mathrm{n}}(\omega)$ as

$$
\mathcal{T}_{\mathrm{n}}(\omega)=\left(1-A_{\mathrm{n}}^{*} \frac{2 J_{1}\left(k_{\mathrm{n}} r_{b}\right)}{k_{\mathrm{n}} r_{b} K_{\mathrm{n}}}\right) .
$$

On the other hand, the second term of Eq. (2) is the force exerted by the wetting fluid phase, whose pressure is given by Eq. (A.4), then,

$$
\begin{aligned}
\Delta F_{\mathrm{w}} & =2 \pi \int_{r_{b}}^{r_{c}} p_{\mathrm{w}}(r) r \mathrm{~d} r \\
& =2 \pi \int_{r_{b}}^{r_{c}}\left(\mathcal{B}_{\mathrm{w}} T_{0}(r)+\frac{C_{\mathrm{w}}}{k_{\mathrm{w}}^{2}}\right) r \mathrm{~d} r \\
& =\left.2 \pi\left(\mathcal{B}_{\mathrm{w}} \frac{T_{1}(r) r}{k_{\mathrm{w}}}+\frac{C_{\mathrm{w}} r^{2}}{2 k_{\mathrm{w}}^{2}}\right)\right|_{r_{b}} ^{r_{c}},
\end{aligned}
$$

where, by definition $T_{1}\left(r_{c}\right)=0$. Then,

$$
\begin{aligned}
\Delta F_{\mathrm{w}} & =\left[2 \pi r_{c}^{2}\left(\mathcal{B}_{\mathrm{w}}^{*} \frac{T_{1}\left(r_{c}\right)}{r_{c} k_{\mathrm{w}} K_{\mathrm{w}}}-\frac{1}{2}\right)-2 \pi r_{b}^{2}\left(\mathcal{B}_{\mathrm{w}}^{*} \frac{T_{1}\left(r_{b}\right)}{r_{b} k_{\mathrm{w}} K_{\mathrm{w}}}-\frac{1}{2}\right)\right] \frac{\Delta h K_{\mathrm{w}}}{h_{0}} \\
& =-\pi\left(r_{c}^{2}-r_{b}^{2}\right)\left[\frac{r_{c}^{2}}{\left(r_{c}^{2}-r_{b}^{2}\right)}-\frac{r_{b}^{2}}{\left(r_{c}^{2}-r_{b}^{2}\right)}\left(1-\mathcal{B}_{\mathrm{w}}^{*} \frac{2 T_{1}\left(r_{b}\right)}{r_{b} k_{\mathrm{w}} K_{\mathrm{w}}}\right)\right] \frac{\Delta h K_{\mathrm{w}}}{h_{0}} \\
& =-\pi\left(r_{c}^{2}-r_{b}^{2}\right) \mathcal{T}_{\mathrm{w}}(\omega) \frac{\Delta h K_{\mathrm{w}}}{h_{0}} \\
& =-\pi r_{c}^{2} S_{\mathrm{w}} \mathcal{T}_{\mathrm{w}}(\omega) \frac{\Delta h K_{\mathrm{w}}}{h_{0}}
\end{aligned}
$$

where we have defined $\mathcal{T}_{\mathrm{w}}(\omega)$ as

$$
\mathcal{T}_{\mathrm{w}}(\omega)=\left[\frac{r_{c}^{2}}{\left(r_{c}^{2}-r_{b}^{2}\right)}-\frac{r_{b}^{2}}{\left(r_{c}^{2}-r_{b}^{2}\right)}\left(1-\mathcal{B}_{\mathrm{w}}^{*} \frac{2 T_{1}\left(r_{b}\right)}{r_{b} k_{\mathrm{w}} K_{\mathrm{w}}}\right)\right] .
$$

\section{APPENDIX B: ANISOTROPIC GASSMANN'S EQUATIONS}

In order to obtain the saturated response of the above described VTI dry medium, we can employ Gassmann (1951) fluid substitution equations for anisotropic porous media, which state

$$
c_{i j}^{\mathrm{sat}}=c_{i j}^{\mathrm{dry}}+\alpha_{i} \alpha_{j} M,
$$

where $\alpha_{i}$ are the effective Biot-Willis stress coefficients given by

$$
\alpha_{m}=1-\frac{\sum_{n=1}^{3} c_{m n}^{\mathrm{dry}}}{3 K_{s}}
$$

and $M$ is the analogue of Gassmann's pore space modulus defined as

$$
M=K_{s}\left[\left(1-\frac{\hat{K}}{K_{s}}\right)-\phi\left(1-\frac{K_{s}}{K_{f}}\right)\right]^{-1}
$$


The generalised bulk modulus is given by

$$
\hat{K}=\frac{1}{9} \sum_{i=1}^{3} \sum_{j=1}^{3} c_{i j} .
$$

As previously mentioned, in this work we are dealing with a VTI medium and, hence, Eq. (B.2) yields

$$
\begin{gathered}
\alpha_{1}=1-\frac{c_{11}+2 c_{13}}{3 K_{s}}, \\
\alpha_{2}=\alpha_{3}=1-\frac{c_{13}+c_{23}+c_{33}}{3 K_{s}}, \\
\text { and } \alpha_{4}=\alpha_{5}=\alpha_{6}=0 .
\end{gathered}
$$

\section{APPENDIX C: POINTER'S LOW-FREQUENCY MODEL FOR PARTIALLY SATURATED CRACKS}

Expressions for the overall stiffnesses of a material containing partially saturated aligned cracks are given by Pointer et al. (2000). This model is valid only at relatively low frequencies and leads to attenuation estimates that are linear in frequency and non-dispersive body wave velocities. In the context of Hudson (1981) equations, which are summarised in subsection 2.5, Pointer et al. (2000) proposes the following parameterisation:

$U_{3}=\frac{4\left(\lambda_{s}+2 \mu_{s}\right)}{3\left(\lambda_{s}+\mu_{s}\right)} \frac{1}{1+\mathcal{K}_{1}}-\frac{\pi}{3} \frac{i \omega \mu}{\alpha}\left(U^{s}\right)^{2}\left[\left(\frac{1}{K_{\mathrm{w}}}-\frac{1}{K_{\mathrm{n}}}\right) /\left(\frac{S_{\mathrm{w}}}{K_{\mathrm{w}}}+\frac{\left(1-S_{\mathrm{w}}\right)}{K_{\mathrm{n}}}\right)\right]^{2}\left[\eta_{\mathrm{w}} F_{\mathrm{w}}+\eta_{\mathrm{n}} F_{\mathrm{n}}\right]$,

where

$$
\begin{aligned}
& U^{s}=\frac{2}{\mu \pi \alpha}\left(\frac{\lambda_{s}+2 \mu_{s}}{\lambda_{s}+\mu_{s}}\right) /\left(1+\mathcal{K}_{1}\right), \\
& \mathcal{K}_{1}=\frac{1}{\pi \alpha \mu_{s}} \frac{\left(\lambda_{s}+2 \mu_{s}\right)}{\left(\lambda_{s}+\mu_{s}\right)}\left(\frac{S_{\mathrm{w}}}{K_{\mathrm{w}}}+\frac{\left(1-S_{\mathrm{w}}\right)}{K_{\mathrm{n}}}\right)^{-1}, \\
& F_{\mathrm{w}}=\left(1-S_{\mathrm{w}}\right)^{2} \int_{\left(1-S_{\mathrm{w}}\right)^{1 / 3}}^{1} \frac{\left(y^{3}-1\right)^{2}}{y^{2}\left(1-y^{2}\right)} \mathrm{d} y, \\
& F_{\mathrm{n}}=S_{\mathrm{w}}^{2} \int_{0}^{\left(1-S_{\mathrm{w}}\right)^{1 / 3}} \frac{y^{4}}{1-y^{2}} \mathrm{~d} y .
\end{aligned}
$$

\section{REFERENCES}

Abramowitz, M. \& Stegun, I. A., 1965. Handbook of Mathematical Functions: With Formulas, Graphs and Mathematical Tables, Dover Publications, Mineola, USA.

Alkhimenkov, Y., Caspari, E., Gurevich, B., Barbosa, N. D., Glubokovskikh, S., Hunziker, J., \& Quintal, B., 
2020. Frequency-dependent attenuation and dispersion caused by squirt flow: Three-dimensional numerical study, Geophysics, 85(3), MR129-MR145.

Andrew, M., Bijeljic, B., \& Blunt, M. J., 2014. Pore-scale contact angle measurements at reservoir conditions using x-ray microtomography, Adv. Water Resour., 68, 24-31.

Batzle, M., Han, D.-h., \& Castagna, J., 1996. Attenuation and velocity dispersion at seismic frequencies, in SEG Tech. Program Expanded Abstr., vol. 15, pp. 1687-1690, Society of Exploration Geophysicists.

Beresnev, I. A., Vigil, R. D., Li, W., Pennington, W. D., Turpening, R. M., Iassonov, P. P., \& Ewing, R. P., 2005. Elastic waves push organic fluids from reservoir rock, Geophys. Res. Lett., 32(13).

Berryman, J. G., Pride, S. R., \& Wang, H. F., 2002. A differential scheme for elastic properties of rocks with dry or saturated cracks, Geophys. J. Int., 151(2), 597-611.

Bishop, A. W. \& Blight, G., 1963. Some aspects of effective stress in saturated and partly saturated soils, Geotechnique, 13(3), 177-197.

Blunt, M. J., 2017. Multiphase flow in permeable media: A pore-scale perspective, Cambridge University Press, Cambridge, UK.

Bourbié, T., Coussy, O., \& Zinszner, B., 1987. Acoustics of porous media, Institut français du pétrole publications, Paris, France.

Brie, A., Pampuri, F., Marsala, A., Meazza, O., et al., 1995. Shear sonic interpretation in gas-bearing sands, in SPE Annual Technical Conference, p. 701-710, Society of Petroleum Engineers.

Budiansky, B. \& O'Connell, R. J., 1976. Elastic moduli of a cracked solid, Int. J. Solids Struc., 12(2), 81-97.

Cadoret, T., Mavko, G., \& Zinszner, B., 1998. Fluid distribution effect on sonic attenuation in partially saturated limestones, Geophysics, 63, 154-160.

Chapman, S., Tisato, N., Quintal, B., \& Holliger, K., 2016. Seismic attenuation in partially saturated Berea sandstone submitted to a range of confining pressures, J. Geophys. Res., 121(3), 1664-1676.

Chapman, S., Borgomano, J. V., Yin, H., Fortin, J., \& Quintal, B., 2019. Forced oscillation measurements of seismic wave attenuation and stiffness moduli dispersion in glycerine-saturated berea sandstone, Geophys. Prospect., 67(4), 956-968.

Cheng, C., 1993. Crack models for a transversely isotropic medium, J. Geophys. Res.: Solid Earth, 98(B1), $675-684$

Crampin, S., 1978. Seismic-wave propagation through a cracked solid: polarization as a possible dilatancy diagnostic, Geophys. J. Int., 53(3), 467-496.

Eshelby, J. D., 1957. The determination of the elastic field of an ellipsoidal inclusion, and related problems, $P$. Roy. Soc. London Ser. A, 241(1226), 376-396.

Fortin, J., Pimienta, L., Guéguen, Y., Schubnel, A., David, E., \& Adelinet, M., 2014. Experimental results on the combined effects of frequency and pressure on the dispersion of elastic waves in porous rocks, The Leading Edge, 33(6), 648-654.

Garbin, H. \& Knopoff, L., 1973. The compressional modulus of a material permeated by a random distribution of circular cracks, Q. Appl. Math., 30(4), 453-464. 
Garbin, H. \& Knopoff, L., 1975a. The shear modulus of a material permeated by a random distribution of free circular cracks, Q. Appl. Math., 33(3), 296-300.

Garbin, H. \& Knopoff, L., 1975b. Elastic moduli of a medium with liquid-filled cracks, Q. Appl. Math., 33(3), 301-303.

Gassmann, F., 1951. Über die Elastizität poröser Medien, Vierteljahresschr. Naturforsch. Ges. Zürich, 96, $1-23$.

Guo, J. \& Gurevich, B., 2020. Effects of coupling between wave-induced fluid flow and elastic scattering on p-wave dispersion and attenuation in rocks with aligned fractures, J. Geophys. Res.: Solid Earth, 125(3), e2019JB018685.

Gurevich, B., Makarynska, D., de Paula, O. B., \& Pervukhina, M., 2010. A simple model for squirt-flow dispersion and attenuation in fluid-saturated granular rocks, Geophysics, 75(6), N109-N120.

Hadley, K., 1976. Comparison of calculated and observed crack densities and seismic velocities in westerly granite, J. Geophys. Res., 81(20), 3484-3494.

Henyey, F. S. \& Pomphrey, N., 1982. Self-consistent elastic moduli of a cracked solid, Geophys. Res. Lett., 9(8), 903-906.

Hudson, J., 1980. Overall properties of a cracked solid, Math. Proc. Cambridge Philos. Soc., 88(02), 371-384.

Hudson, J., 1988. Seismic wave propagation through material containing partially saturated cracks, Geophys. J. Int., 92(1), 33-37.

Hudson, J. A., 1981. Wave speeds and attenuation of elastic waves in material containing cracks, Geophys. $J$. Int., 64(1), 133-150.

Johnson, D. L., 2001. Theory of frequency dependent acoustics in patchy-saturated porous media, J. Acoust. Soc. Am., 110, 682-694.

Mavko, G., Mukerji, T., \& Dvorkin, J., 2009. The Rock Physics Handbook: Tools for Seismic Analysis of Porous Media, Cambridge University Press, Cambridge, UK.

Mavko, G. M. \& Nur, A., 1979. Wave attenuation in partially saturated rocks, Geophysics, 44, 161-178, doi:10.1190/1.1440958.

Miksis, M. J., 1988. Effects of contact line movement on the dissipation of waves in partially saturated rocks, J. Geophys. Res.: Solid Earth, 93(B6), 6624-6634.

Müller, T. M., Gurevich, B., \& Lebedev, M., 2010. Seismic wave attenuation and dispersion resulting from wave-induced flow in porous rocks - a review, Geophysics, 75, 147-163.

Murphy, W. F., Winkler, K. W., \& Kleinberg, R. L., 1986. Acoustic relaxation in sedimentary rocks: Dependence on grain contacts and fluid saturation, Geophysics, 51(3), 757-766.

Palmer, I. \& Traviolia, M., 1980. Attenuation by squirt flow in undersaturated gas sands, Geophysics, 45(12), 1780-1792.

Papageorgiou, G., Amalokwu, K., \& Chapman, M., 2016. Theoretical derivation of a brie-like fluid mixing law, Geophys. Prospect., 64, 1048-1053.

Pointer, T., Liu, E., \& Hudson, J. A., 2000. Seismic wave propagation in cracked porous media, Geophys. J. 
Int., 142(1), 199-231.

Pride, S. R., Flekkøy, E. G., \& Aursjø, O., 2008. Seismic stimulation for enhanced oil recovery, Geophysics, 73(5), O23-O35.

Quintal, B., Rubino, J. G., Caspari, E., \& Holliger, K., 2016. A simple hydromechanical approach for simulating squirt-type flow, Geophysics, 81(4), D335-D344.

Quintal, B., Caspari, E., Holliger, K., \& Steeb, H., 2019. Numerically quantifying energy loss caused by squirt flow, Geophys. Prospect., 67(8), 2196-2212.

Rozhko, A. Y. \& Bauer, A., 2019. Contact line friction and surface tension effects on seismic attenuation and effective bulk moduli in rock with a partially saturated crack, Geophys. Prospect., 67(4), 913-934.

Rubino, J. G. \& Holliger, K., 2012. Seismic attenuation and velocity dispersion in heterogeneous partially saturated porous rocks, Geophys. J. Int., 188, 1088-1102.

Rubino, J. G., Caspari, E., Müller, T. M., Milani, M., Barbosa, N. D., \& Holliger, K., 2016. Numerical upscaling in 2-D heterogeneous poroelastic rocks: Anisotropic attenuation and dispersion of seismic waves, J. Geophys. Res.: Solid Earth, 121(9), 6698-6721.

Sarout, J., Cazes, E., Delle Piane, C., Arena, A., \& Esteban, L., 2017. Stress-dependent permeability and wave dispersion in tight cracked rocks: Experimental validation of simple effective medium models, J. Geophys. Res.: Solid Earth, 122(8), 6180-6201.

Solazzi, S. G., Guarracino, L., Rubino, J. G., Müller, T. M., \& Holliger, K., 2017. Modeling forced imbibition processes and the associated seismic attenuation in heterogeneous porous rocks, J. Geophys. Res. Solid Earth, 122(11), 9031-9049.

Solazzi, S. G., Guarracino, L., Rubino, J. G., \& Holliger, K., 2019. Saturation hysteresis effects on the seismic signatures of partially saturated heterogeneous porous rocks, J. Geophys. Res. Solid Earth, 124(11), 1131611335 .

Subramaniyan, S., Quintal, B., Madonna, C., \& Saenger, E. H., 2015. Laboratory-based seismic attenuation in fontainebleau sandstone: Evidence of squirt flow, J. Geophys. Res. Solid Earth, 120(11), 7526-7535.

Takamura, K., Fischer, H., \& Morrow, N. R., 2012. Physical properties of aqueous glycerol solutions, J. Petrol. Sci. Eng., 98, 50-60.

Tisato, N. \& Quintal, B., 2013. Measurements of seismic attenuation and transient fluid pressure in partially saturated Berea sandstone: Evidence of fluid flow on the mesoscopic scale, Geophys. J. Int., 195, 342-351.

Walsh, J., 1995. Seismic attenuation in partially saturated rock, J. Geophys. Res., 100(B8), 15407-15424.

White, J. E., 1975. Computed seismic speeds and attenuation in rocks with partial gas saturation, Geophysics, 40, 224-232.

White, J. E., Mikhaylova, N. G., \& Lyakhovitsky, F. M., 1975. Low-frequency seismic waves in fluid-saturated layered rocks, Phys. Solid Earth, 11, 654-659.

Yin, C. S., Batzle, M. L., \& Smith, B. J., 1992. Effects of partial liquid/gas saturation on extensional wave attenuation in Berea sandstone, Geophys. Res. Lett., 19(13), 1399-1402. 